\title{
Kinetic Resolution of Alcohols USing a 1,2-DihydRoimidazo[1,2-A]Quinoline Enantioselective ACylation Catalyst
}

\author{
Vladimir B. Birman* and Hui Jiang \\ A contribution from the Department of Chemistry, Washington University, Campus Box 1134, \\ One Brookings Drive, Saint Louis, Missouri 63130
}

SUPPORTING INFORMATION: Experimental procedures (9 pages), ${ }^{1} \mathrm{H}$ NMR and ${ }^{13} \mathrm{C}$ NMR spectra (32 pages).

\section{Experimental Part.}

General. All reagents were obtained commercially and used as received unless otherwise specified. First-generation catalyst $\mathrm{CF}_{3}$-PIP was prepared as previously described. ${ }^{1}$ Preparations of catalyst precursors requiring elevated pressures were carried out in thick-wall pressure tubes with Teflon screwcaps available from Chemglass. ( \pm -1-Phenylethanol (12), $( \pm)-1$-phenyl-1propanol (13), ( \pm )-2,2-dimethyl-1-phenyl-1-propanol (15) and $( \pm)$ - $\square$-methyl-2naphthalenemethanol (16) are available from Aldrich. ( \pm )-E-4-Phenyl-but-3-en-2-ol (6), $( \pm)$-E2-methyl-5-phenyl-pent-4-en-3-ol (7), ( \pm )-E-3-methyl-4-phenyl-but-3-en-2-ol (8), $( \pm)$-E-2,4dimethyl-5-phenyl-pent-4-en-3-ol (9) were prepared by addition of methyl- or isopropylGrignard reagents to the corresponding commercially available aldehydes. $( \pm)$-2-Methyl-1-

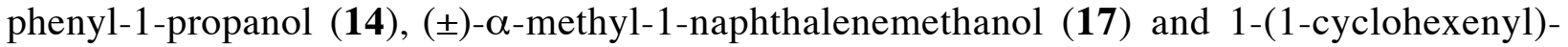
ethanol ${ }^{9}(\mathbf{1 8})$ were prepared by borohydride reduction of the corresponding commercially available ketones. ( \pm )-E-4-(o-Methoxyphenyl)-but-3-en-2-ol (10) ${ }^{10 a}$ was prepared by borohydride reduction of the corresponding ketone obtained by aldol condensation of oanisaldehyde with acetone. $.^{10 \mathrm{~b}}( \pm)-1-\left(\mathrm{Chrom}-3\right.$-en-3-yl)-ethanol $(\mathbf{1 1})^{1 \mathrm{ala}}$ was prepared by borohydride reduction of the corresponding ketone obtained from salicylaldehyde and methyl vinyl ketone. ${ }^{11 \mathrm{~b}}$ Chloroform (OmniSolv® grade, stabilized with nonpolar hydrocarbons) was obtained from EM Science. Solvents used for chromatography were ACS or HPLC grade, as appropriate. Reactions were monitored by thin layer chromatography (TLC) using EM Science 60F silica gel plates. Flash column chromatography was performed over ICN Ecochrom silica gel (32-63 $\square \mathrm{m})$. Deuterated chloroform was obtained from Aldrich. ${ }^{1} \mathrm{H}$ NMR and ${ }^{13} \mathrm{C}$ NMR spectra were recorded on a Unity $300 \mathrm{MHz}$ Varian spectrometer. The chemical shifts are reported as $\mathrm{Q}$ values (ppm) relative to TMS using residual $\mathrm{CHCl}_{3}$ peak $(7.26 \mathrm{ppm})$ as the reference. High-Resolution mass spectral analyses were performed at Washington University MS Center on a Kratos MS-50TA spectrometer using electron impact method (EI). Melting points were measured on a Mel-Temp II capillary melting point apparatus. Infrared spectra were recorded on a Perkin-Elmer Spectrum Bx FTIR spectrophotometer using potassium bromide plates. Optical rotations were measured at room temperature and $589 \mathrm{~nm}$ wavelength using a Perkin-Elmer 241 polarimeter. HPLC analyses were performed on a Shimadzu LC system using isopropanol-hexanes mobile phase at a flow rate of $1 \mathrm{~mL} / \mathrm{min}$ and $\mathrm{UV}$ detection at $254 \mathrm{~nm}$ as previously described. ${ }^{1}$ The absolute chirality of the unreacted alcohols obtained by kinetic resolution was either assigned by comparison of the sign of optical rotation with literature data or determined by Mosher ester analysis. 


\section{CATAlyst PREPARATion.}

\section{(R)-N-(6-chloroquinolinyl-2)-2-Hydroxy-1-phenylethylamine (5)}

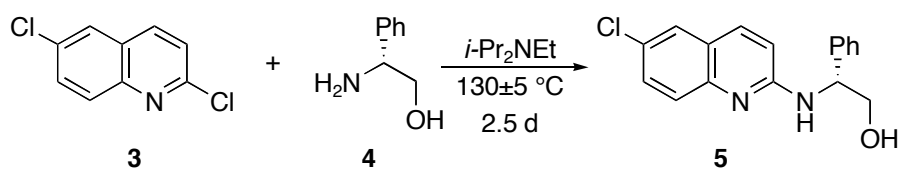

A 15-mL pressure tube charged with 2,6-dichloroquinoline 3 (1.352 g, $6.83 \mathrm{mmol})$, (R)phenylglycinol 4 (0.964 g, $7.03 \mathrm{mmol})$, N, N-diisopropylethylamine $(0.991 \mathrm{~g}, 7.67 \mathrm{mmol})$ and a stir bar was flushed with nitrogen several times, stoppered and immersed into an oil bath kept at $130 \pm 5{ }^{\circ} \mathrm{C}$ for 2.5 days. The tube was allowed to cool to room temperature and the content was diluted with $\mathrm{CH}_{2} \mathrm{Cl}_{2}$, which led to precipitation of a white solid. The mixture was washed with saturated aqueous $\mathrm{NH}_{4} \mathrm{Cl}$ to remove $\mathrm{N}, \mathrm{N}$-diisopropylethylamine and then with saturated aqueous $\mathrm{NaHCO}_{3}$. The solution was dried over $\mathrm{Na}_{2} \mathrm{SO}_{4}$ and evaporated to afford $1.770 \mathrm{~g}$ of the crystalline product $(87 \%)$, which was sufficiently pure for the next step. If necessary, the product can be chromatographed (6\% isopropanol, $0.8 \%$ triethylamine in hexanes).

${ }^{1} \mathbf{H}$ NMR $\left(300 \mathrm{MHz}, \mathrm{CDCl}_{3}\right) \square 7.73(\mathrm{~d} ; \mathrm{J}=9 \mathrm{~Hz} ; 1 \mathrm{H}), 7.62(\mathrm{~d} ; \mathrm{J}=8.7 \mathrm{~Hz} ; 1 \mathrm{H}), 7.62$ (d; J = 8.7 $\mathrm{Hz} ; 1 \mathrm{H}), 7.55(\mathrm{~d} ; \mathrm{J}=2.2 \mathrm{~Hz} ; 1 \mathrm{H}), 7.48\left(\mathrm{dd} ; \mathrm{J}_{1}=9 \mathrm{~Hz}, \mathrm{~J}_{2}=2.2 \mathrm{~Hz} ; 1 \mathrm{H}\right), 7.28 \sim 7.45(\mathrm{~m} ; 5 \mathrm{H}), 6.67$ $(\mathrm{d} ; \mathrm{J}=9 \mathrm{~Hz} ; 1 \mathrm{H}), 5.85(\mathrm{~s} ; 1 \mathrm{H}), 5.16\left(\mathrm{dt} ; \mathrm{J}_{1}=7.4 \mathrm{~Hz}, \mathrm{~J}_{2}=3.6 \mathrm{~Hz} ; 1 \mathrm{H}\right), 4.05\left(\mathrm{dd} ; \mathrm{J}_{1}=11.3 \mathrm{~Hz}, \mathrm{~J}_{2}=\right.$ $7.4 \mathrm{~Hz} ; 1 \mathrm{H}), 3.00\left(\mathrm{dd} ; \mathrm{J}_{1}=11.3 \mathrm{~Hz}, \mathrm{~J}_{2}=3.6 \mathrm{~Hz} ; 1 \mathrm{H}\right) ;{ }^{13} \mathbf{C} \mathbf{N M R}\left(75 \mathrm{MHz}, \mathrm{CDCl}_{3}\right) \square 156.7$, $144.8,140.0,137.0,130.5,129.0,128.05,127.98,126.88,126.81,126.2,123.9,113.0,68.3$, 59.8; IR (film, $\mathrm{cm}^{-1}$ ) 3316 (br) 1618.7, 1498.6, 1398.4; MS: HR-EI calculated for $\mathrm{C}_{17} \mathrm{H}_{15} \mathrm{ClN}_{2} \mathrm{O}$ $\left(\mathrm{M}^{+}\right) \mathrm{m} / \mathrm{z}: 298.0873$, found: $298.0904 ; \mathbf{m p ~} 172-174{ }^{\circ} \mathrm{C}$; [ $]_{\mathrm{D}}=-154^{\circ}(\mathrm{c}=0.26, \mathrm{MeOH})$.

\section{(R)-7-Chloro-2-phenyl-2, 3-dihydroimidazo[1,2-a]quinoline (Cl-PIQ, 2)}

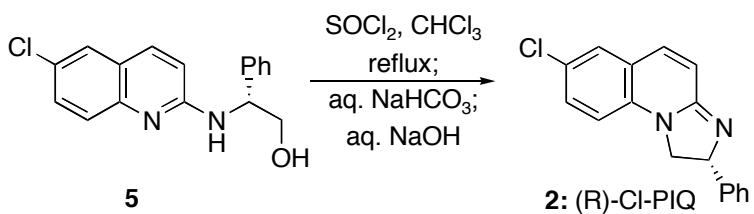

A solution of $5(0.365 \mathrm{~g}, 1.22 \mathrm{mmol})$ in $6.4 \mathrm{~mL}$ of $\mathrm{CHCl}_{3}$ was treated with $\mathrm{SOCl}_{2}(0.23 \mathrm{~mL}$, $3.15 \mathrm{mmol}$ ), and heated to reflux at $60 \sim 65{ }^{\circ} \mathrm{C}$. After 1.5 hours, the reaction mixture was allowed to cool somewhat, treated with a few drops of $\mathrm{MeOH}$, and then heated again for 10 minutes and allowed to cool to room temperature. The mixture was treated with saturated aqueous $\mathrm{NaHCO}_{3}$ until the aqueous phase reached $\mathrm{pH} 8$, then with $1 \mathrm{M} \mathrm{NaOH}$ to $\mathrm{pH} 14$, and extracted with $\mathrm{CH}_{2} \mathrm{Cl}_{2}$. The organic extract was dried over $\mathrm{Na}_{2} \mathrm{SO}_{4}$, concentrated, and chromatographed $(10 \% \rightarrow 15 \%$ isopropanol, $2 \%$ triethylamine in hexanes) to afford $0.311 \mathrm{~g}$ of yellow oil (91\%), which crystallized on standing.

${ }^{1} \mathbf{H}$ NMR $\left(300 \mathrm{MHz}, \mathrm{CDCl}_{3}\right) \square 7.20-7.40(\mathrm{~m} ; 8 \mathrm{H}), 6.76(\mathrm{~d} ; \mathrm{J}=9.3 \mathrm{~Hz} ; 1 \mathrm{H}), 6.65(\mathrm{~d} ; \mathrm{J}=8.8 \mathrm{~Hz}$; $1 \mathrm{H}), 5.40\left(\mathrm{dd} ; \mathrm{J}_{1}=11.4 \mathrm{~Hz}, \mathrm{~J}_{2}=8.4 \mathrm{~Hz} ; 1 \mathrm{H}\right), 4.40\left(\mathrm{dd} ; \mathrm{J}_{1}=11.4 \mathrm{~Hz}, \mathrm{~J}_{2}=10.3 \mathrm{~Hz} ; 1 \mathrm{H}\right), 3.88(\mathrm{dd}$; $\left.\mathrm{J}_{1}=10.3 \mathrm{~Hz}, \mathrm{~J}_{2}=8.4 \mathrm{~Hz} ; 1 \mathrm{H}\right) ;{ }^{13} \mathbf{C} \mathbf{N M R}\left(75 \mathrm{MHz}, \mathrm{CDCl}_{3}\right) \square 156.2,143.9,137.5,135.8,130.2$, 128.7, 127.5, 127.4, 126.6, 125.4, 122.2, 118.4, 112.7, 67.7, 54.1; IR (film, $\mathrm{cm}^{-1}$ ) 1640.3, 1568.9, 
1433.9; MS: HR-EI calculated for $\mathrm{C}_{17} \mathrm{H}_{13} \mathrm{ClN}_{2}\left(\mathrm{M}^{+}\right) \mathrm{m} / \mathrm{z}$ : 280.0767, found: 280.0770 ; mp 134$136{ }^{\circ} \mathrm{C}$ (from $\mathrm{Et}_{2} \mathrm{O}$-hexanes); [ $]_{\mathrm{D}}=+746^{\circ}(\mathrm{c}=0.27, \mathrm{MeOH})$.

Note: The catalyst recrystallized from $\mathrm{Et}_{2} \mathrm{O}$-hexanes did not show a significant improvement of selectivity compared with the catalyst that was obtained after chromatography (using $\mathbf{1 7}$ as the test substrate). The data reported were obtained with the unrecrystallized catalyst.

\section{KINETIC RESOLUTION EXPERIMENTS.}

Substrates 6-17.

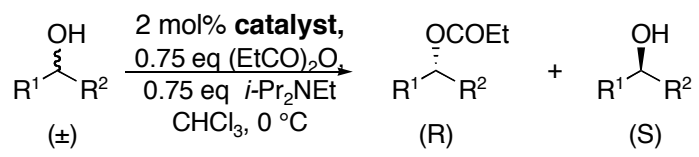

Kinetic resolution experiments with substrates 6-17 were carried out according to the previously published procedure, ${ }^{1}$ except that for the most acid-sensitive substrates $\mathbf{1 0}$ and $\mathbf{1 1}$ the treatment with dilute $\mathrm{HCl}$ during the workup was omitted and the crude mixtures were directly applied to silica gel and chromatographed.

\section{Substrate 18.}
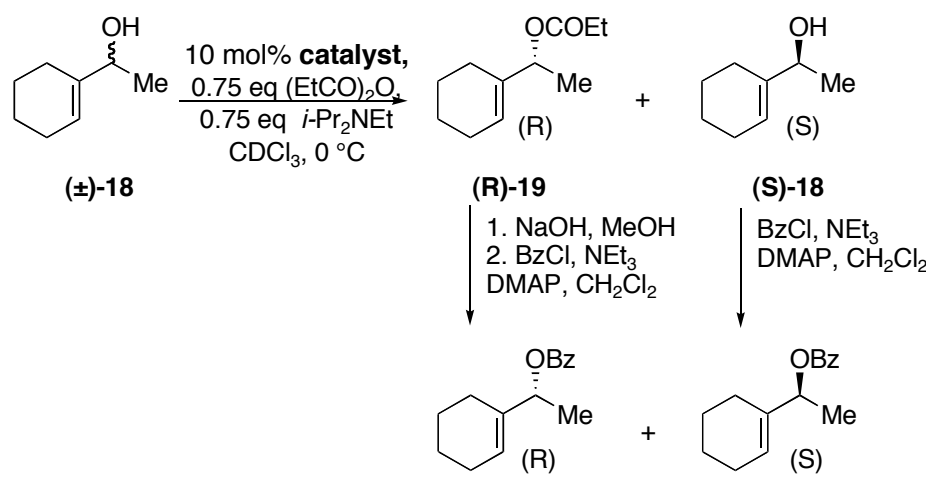

(R)-20

(S)-20

1) The substrate $(63 \mathrm{mg}, 0.50 \mathrm{mmol}), 0.050 \mathrm{mmol}$ of the catalyst and $0.50 \mathrm{~mL}$ of $0.75 \mathrm{M}$ solution of $i$ - $\mathrm{Pr}_{2} \mathrm{NEt}$ in $\mathrm{CDCl}_{3}$ were placed in a one-dram vial. The vial was cooled in an ice bath for 15 minutes before propionic anhydride precooled to $0{ }^{\circ} \mathrm{C}(48 \square \mathrm{L}, 0.375 \mathrm{mmol}$ ) was added. The vial was left in the ice bath. The progress of acylation was checked periodically by withdrawing a small aliquot of the reaction mixture, diluting it with $\mathrm{CDCl}_{3}$ and analyzing by ${ }^{1} \mathrm{H}$ NMR. After reaching conversion over $40 \%$ by NMR, the reaction mixture was quenched by adding $0.5 \mathrm{~mL}$ of methanol and left at $0{ }^{\circ} \mathrm{C}$ for $0.5 \mathrm{~h}$ and at room temperature for $1 \mathrm{~h}$. The mixture was diluted with $\mathrm{CH}_{2} \mathrm{Cl}_{2}$, washed with saturated $\mathrm{NH}_{4} \mathrm{Cl}$, brine, saturated $\mathrm{NaHCO}_{3}$ and brine again and dried over $\mathrm{Na}_{2} \mathrm{SO}_{4}$, and rotary evaporated. The propionate ester and the unreacted alcohol were separated by column chromatography $\left(5 \rightarrow 15 \% \mathrm{Et}_{2} \mathrm{O}\right.$ in hexanes). The ester was hydrolyzed with $2 \mathrm{M}$ aqueous $\mathrm{MeOH}$ for $2 \mathrm{~h}$. The resulting alcohol was isolated by standard aqueous workup and purified by chromatography (short column, $15 \% \mathrm{Et}_{2} \mathrm{O}$ in hexanes). 2) The unreacted alcohol and the alcohol obtained by hydrolysis of the propionate ester were benzoylated to permit their separation on a chiral HPLC column. Each sample of the alcohol 
was dissolved in $0.5 \mathrm{~mL}$ of $\mathrm{CH}_{2} \mathrm{Cl}_{2}$ and treated with $55 \square \mathrm{L}$ of $\mathrm{NEt}_{3}, 2-3 \mathrm{mg}$ of DMAP and $35 \square \mathrm{L}$ of benzoyl chloride at room temperature overnight. The reaction mixture was quenched by adding $15 \square \mathrm{L}$ of ethylenediamine, diluted with $\mathrm{CH}_{2} \mathrm{Cl}_{2}$, and washed with $1 \mathrm{M} \mathrm{HCl}$ and then saturated $\mathrm{NaHCO}_{3}$. The organic phase was dried over $\mathrm{Na}_{2} \mathrm{SO}_{4}$ and rotary evaporated. The semisolid residue was taken up in the eluent and chromatographed (4\% $\mathrm{Et}_{2} \mathrm{O}$ in hexanes). The resulting benzoate $\mathbf{2 0}$ was analyzed by HPLC using CHIRALPAK AD analytical column (4.6x250 mm, Chiral Technologies, Inc.) and 0.5\% isopropanol in hexane as the mobile phase.

\section{Spectral data for previously uncharacterized substrates and esters.}

Propionate of 6: ${ }^{1} \mathbf{H}$ NMR $\left(300 \mathrm{MHz}, \mathrm{CDCl}_{3}\right) \square 7.40-7.24(\mathrm{~m} ; 5 \mathrm{H}), 6.61(\mathrm{~d} ; \mathrm{J}=15.9 \mathrm{~Hz} ; 1 \mathrm{H})$, $6.20\left(\mathrm{dd} ; \mathrm{J}_{1}=15.9 \mathrm{~Hz}, \mathrm{~J}_{2}=6.6 \mathrm{~Hz} ; 1 \mathrm{H}\right), 5.55$ (quintet; $\left.\mathrm{J}=6.6 \mathrm{~Hz} ; 1 \mathrm{H}\right), 2.36\left(q d ; \mathrm{J}_{1}=7.4 \mathrm{~Hz}, \mathrm{~J}_{2}=\right.$ $0.8 \mathrm{~Hz} ; 2 \mathrm{H}), 1.42(\mathrm{~d} ; \mathrm{J}=6.6 \mathrm{~Hz} ; 3 \mathrm{H}), 1.16(\mathrm{t} ; \mathrm{J}=7.4 \mathrm{~Hz} ; 3 \mathrm{H}) ;{ }^{13} \mathbf{C} \mathbf{N M R}\left(75 \mathrm{MHz}, \mathrm{CDCl}_{3}\right)$ $\square$ 173.7, 136.3, 131.4, 128.9, 128.5, 127.8, 126.5, 70.7, 27.9, 20.3, 9.1; MS: HR-EI calculated for $\mathrm{C}_{13} \mathrm{H}_{16} \mathrm{O}_{2}\left(\mathrm{M}^{+}\right) \mathrm{m} / \mathrm{z}: 204.1150$, measured m/z: 204.1150; IR $\left(\mathrm{KBr}, \mathrm{cm}^{-1}\right)$ 1734.0, 1187.3.

Propionate of 7: ${ }^{1} \mathbf{H}$ NMR (300 MHz, $\left.\mathrm{CDCl}_{3}\right) \square 7.40-7.23(\mathrm{~m} ; 5 \mathrm{H}), 6.59(\mathrm{~d} ; \mathrm{J}=15.9 \mathrm{~Hz} ; 1 \mathrm{H})$, $6.12\left(\mathrm{dd} ; \mathrm{J}_{1}=15.9 \mathrm{~Hz}, \mathrm{~J}_{2}=6.9 \mathrm{~Hz} ; 1 \mathrm{H}\right), 5.22(\mathrm{t} ; \mathrm{J}=6.9 \mathrm{~Hz} ; 1 \mathrm{H}), 2.37$ (apparent qd; $\mathrm{J}_{1}=7.6 \mathrm{~Hz}$, $\left.\mathrm{J}_{2}=1.0 \mathrm{~Hz} ; 2 \mathrm{H}\right), 1.96$ (octet; J =6.9 Hz; 1H), $1.16(\mathrm{t} ; \mathrm{J}=7.6 \mathrm{~Hz} ; 3 \mathrm{H}), 0.96(\mathrm{~d} ; \mathrm{J}=6.9 \mathrm{~Hz} ; 3 \mathrm{H})$, $0.95(\mathrm{~d} ; \mathrm{J}=6.9 \mathrm{~Hz} ; 3 \mathrm{H}) ;{ }^{13} \mathbf{C} \mathbf{N M R}\left(75 \mathrm{MHz}, \mathrm{CDCl}_{3}\right) \square 173.8,136.5,133.2,128.5,127.8,126.5$, 126.3, 79.1, 32.4, 27.9, 18.2, 9.2; MS: HR-EI calculated for $\mathrm{C}_{15} \mathrm{H}_{20} \mathrm{O}_{2}\left(\mathrm{M}^{+}\right) \mathrm{m} / \mathrm{z}$ : 232.1463, measured m/z: 232.1457; IR (KBr) 2964.8, 1732.5, $1185.7 \mathrm{~cm}^{-1}$.

Propionate of 8: ${ }^{1} \mathbf{H}$ NMR $\left(300 \mathrm{MHz}, \mathrm{CDCl}_{3}\right) \square 7.36-7.19(\mathrm{~m} ; 5 \mathrm{H}), 6.52(\mathrm{~s} ; 1 \mathrm{H}), 5.43$ (q; J =6.7 $\mathrm{Hz} ; 1 \mathrm{H}), 2.36(\mathrm{q} ; \mathrm{J}=7.7 \mathrm{~Hz} ; 2 \mathrm{H}), 1.88(\mathrm{~s} ; 3 \mathrm{H}), 1.41(\mathrm{~d} ; \mathrm{J}=6.7 \mathrm{~Hz} ; 3 \mathrm{H}), 1.16(\mathrm{t} ; \mathrm{J}=7.7 \mathrm{~Hz} ; 3 \mathrm{H})$; ${ }^{13}$ C NMR (75 MHz, $\left.\mathrm{CDCl}_{3}\right) \square$ 173.6, 137.4, 137.2, 129.0, 128.0, 126.5, 126.2, 75.1, 27.9, 19.3, 13.8, 9.14; MS: HR-EI calculated for $\mathrm{C}_{14} \mathrm{H}_{18} \mathrm{O}_{2}\left(\mathrm{M}^{+}\right) \mathrm{m} / \mathrm{z}: 218.1307$, measured m/z: 218.1312; IR (KBr) 2981.6, 1736.7, 1186.9 .

Propionate of 9: ${ }^{1} \mathbf{H}$ NMR $\left(300 \mathrm{MHz}, \mathrm{CDCl}_{3}\right) \square 7.35-7.21(\mathrm{~m} ; 5 \mathrm{H}), 6.49(\mathrm{~s} ; 1 \mathrm{H}), 4.99$ (d; J = 8.2 $\mathrm{Hz} ; 1 \mathrm{H}), 2.37$ (q; J = 7.5 Hz; 2H), 2.03 (doublet of septets; $\mathrm{J}_{1}=8.2 \mathrm{~Hz} ; \mathrm{J}_{2}=6.7 \mathrm{~Hz} ; 1 \mathrm{H}$ ), 1.84 (s; $3 \mathrm{H}), 1.17(\mathrm{t} ; \mathrm{J}=7.5 \mathrm{~Hz} ; 3 \mathrm{H}), 0.96(\mathrm{~d} ; \mathrm{J}=6.7 \mathrm{~Hz} ; 3 \mathrm{H}), 0.91(\mathrm{~d} ; \mathrm{J}=6.7 \mathrm{~Hz} ; 3 \mathrm{H}) ;{ }^{13} \mathbf{C} \mathbf{N M R}(75$ $\left.\mathrm{MHz}, \mathrm{CDCl}_{3}\right) \square 173.7,137.3,135.4,129.0,128.5,128.0,126.5,84.3,30.2,27.9,19.1,18.5$, 13.8, 9.2; MS: HR-EI calculated for $\mathrm{C}_{16} \mathrm{H}_{22} \mathrm{O}_{2}\left(\mathrm{M}^{+}\right) \mathrm{m} / \mathrm{z}$ : 246.1620, measured m/z: 246.1620; IR (KBr) 2964.3, 1737.7, $1184.0 \mathrm{~cm}^{-1}$.

Alcohol 10: ${ }^{1} \mathbf{H}$ NMR (300 MHz, $\left.\mathrm{CDCl}_{3}\right) \square 7.44\left(\mathrm{dd} ; \mathrm{J}_{1}=7.7 \mathrm{~Hz}, \mathrm{~J}_{2}=1.6 \mathrm{~Hz} ; 1 \mathrm{H}\right), 7.23\left(\mathrm{td} ; \mathrm{J}_{1}=\right.$ $\left.7.4 \mathrm{~Hz}, \mathrm{~J}_{2}=1.6 \mathrm{~Hz} ; 1 \mathrm{H}\right), 6.95-6.85(\mathrm{~m} ; 3 \mathrm{H}), 6.27\left(\mathrm{dd} ; \mathrm{J}_{1}=15.9 \mathrm{~Hz}, \mathrm{~J}_{2}=6.3 \mathrm{~Hz} ; 1 \mathrm{H}\right), 4.50(\mathrm{q} ; \mathrm{J}=$ $6.3 \mathrm{~Hz} ; 1 \mathrm{H}), 3.85(\mathrm{~s} ; 3 \mathrm{H}), 1.66(\mathrm{~s}, \mathrm{br} ; 1 \mathrm{H}), 1.37(\mathrm{~d} ; \mathrm{J}=6.3 \mathrm{~Hz} ; 3 \mathrm{H}) ;{ }^{13} \mathbf{C} \mathbf{N M R}\left(75 \mathrm{MHz}, \mathrm{CDCl}_{3}\right)$ $\square$ 156.7, 134.2, 128.7, 126.8, 125.6, 124.3, 120.6, 110.8, 69.4, 55.4, 23.3; MS: HR-EI calculated for $\mathrm{C}_{11} \mathrm{H}_{14} \mathrm{O}_{2}\left(\mathrm{M}^{+}\right) \mathrm{m} / \mathrm{z}: 178.0994$, measured m/z: 178.994; IR $\left(\mathrm{KBr}, \mathrm{cm}^{-1}\right) 3356.2$ (br), 1489.1, 1243.4.

Propionate of 10: $\quad{ }^{1} \mathbf{H}$ NMR $\left(300 \mathrm{MHz}, \mathrm{CDCl}_{3}\right) \square 7.43(\mathrm{~d} ; \mathrm{J}=7.7 \mathrm{~Hz} ; 1 \mathrm{H}), 7.23$ (t; J =7.1 Hz; $1 \mathrm{H}), 6.96-6.85(\mathrm{~m} ; 3 \mathrm{H}), 6.22\left(\mathrm{dd} ; \mathrm{J}_{1}=16.2 \mathrm{~Hz}, \mathrm{~J}_{2}=6.7 \mathrm{~Hz} ; 1 \mathrm{H}\right), 5.55$ (quintet; $\mathrm{J}=6.7 \mathrm{~Hz} ; 1 \mathrm{H}$ ), 
$3.84(\mathrm{~s} ; 3 \mathrm{H}), 2.34(\mathrm{q} ; \mathrm{J}=7.4 \mathrm{~Hz} ; 2 \mathrm{H}), 1.41(\mathrm{~d} ; \mathrm{J}=6.7 \mathrm{~Hz} ; 3 \mathrm{H}), 1.15(\mathrm{t} ; \mathrm{J}=7.4 \mathrm{~Hz} ; 3 \mathrm{H}) ;{ }^{13} \mathbf{C}$ NMR $\left(75 \mathrm{MHz}, \mathrm{CDCl}_{3}\right) \square 173.7,156.8,129.4,128.9,126.9,126.2,125.3,120.5,110.8,71.3$, 55.4, 27.9, 20.4, 9.1; MS: HR-EI calculated for $\mathrm{C}_{14} \mathrm{H}_{18} \mathrm{O}_{3}\left(\mathrm{M}^{+}\right) \mathrm{m} / \mathrm{z}: 234.1256$, measured m/z: 234.1248; IR (KBr) 2979.6, $1731.9 \mathrm{~cm}^{-1}$.

Alcohol 11: ${ }^{1} \mathbf{H}$ NMR $\left(300 \mathrm{MHz}, \mathrm{CDCl}_{3}\right) \square 7.11\left(\mathrm{td} ; \mathrm{J}_{1}=7.4 \mathrm{~Hz}, \mathrm{~J}_{2}=1.4 \mathrm{~Hz} ; 1 \mathrm{H}\right), 7.00\left(\mathrm{dd} ; \mathrm{J}_{1}=\right.$ $\left.7.4 \mathrm{~Hz}, \mathrm{~J}_{2}=1.4 \mathrm{~Hz} ; 1 \mathrm{H}\right), 6.87\left(\mathrm{td} ; \mathrm{J}_{1}=7.4 \mathrm{~Hz}, \mathrm{~J}_{2}=1.4 \mathrm{~Hz} ; 1 \mathrm{H}\right), 6.80(\mathrm{~d} ; \mathrm{J}=7.4 \mathrm{~Hz} ; 1 \mathrm{H}), 6.38$ (s; $1 \mathrm{H}), 4.81(\mathrm{~s} ; 2 \mathrm{H}), 4.42(\mathrm{q} ; \mathrm{J}=6.2 \mathrm{~Hz} ; 1 \mathrm{H}), 1.61(\mathrm{~s} ; 1 \mathrm{H}), 1.39(\mathrm{~d} ; \mathrm{J}=6.2 \mathrm{~Hz} ; 3 \mathrm{H}) ;{ }^{13} \mathbf{C}$ NMR $(75$ $\left.\mathrm{MHz}, \mathrm{CDCl}_{3}\right) \square 153.5,137.4,129.0,126.7,122.4,121.5,118.4,115.5,68.6,65.4,21.3$; MS: HR-EI calculated for $\mathrm{C}_{11} \mathrm{H}_{12} \mathrm{O}_{2}\left(\mathrm{M}^{+}\right) \mathrm{m} / \mathrm{z}: 176.0837$, measured $\mathrm{m} / \mathrm{z}: 176.0832 ; \mathbf{I R}\left(\mathrm{KBr}, \mathrm{cm}^{-1}\right)$ 3368.8 (br), 1487.2 .

Propionate of 11: $\quad{ }^{1} \mathbf{H}$ NMR $\left(300 \mathrm{MHz}, \mathrm{CD}_{3} \mathrm{COCD}_{3}\right) \square 7.14-7.06(\mathrm{~m} ; 2 \mathrm{H}), 6.87(\mathrm{t} ; \mathrm{J}=7.4 \mathrm{~Hz}$; $1 \mathrm{H}), 6.75(\mathrm{~d} ; \mathrm{J}=8.0 \mathrm{~Hz} ; 1 \mathrm{H}), 6.49(\mathrm{~s} ; 1 \mathrm{H}), 5.44(\mathrm{q} ; \mathrm{J}=6.4 \mathrm{~Hz} ; 1 \mathrm{H}), 4.80(\mathrm{~d} ; \mathrm{J}=14.4 \mathrm{~Hz} ; 1 \mathrm{H})$, 4.72 (d; J = 14.4 Hz; 1H), 2.36 (q; J = 7.3 Hz; 2H); 1.40 (d; J =6.4 Hz; 3H), 1.09 (t; J = $7.3 \mathrm{~Hz}$; $3 \mathrm{H}) ;{ }^{13} \mathrm{C}$ NMR $\left(75 \mathrm{MHz}, \mathrm{CD}_{3} \mathrm{COCD}_{3}\right) \square 173.7,154.5,135.0,130.0,127.9,123.3,122.3,120.7$, 116.2, 70.7, 66.0, 28.1, 18.8, 9.5; MS: HR-EI calculated for $\mathrm{C}_{14} \mathrm{H}_{16} \mathrm{O}_{3}\left(\mathrm{M}^{+}\right) \mathrm{m} / \mathrm{z}$ : 232.1099, measured m/z: 232.1097; IR (KBr) 2980.2, 1735.0, $1185.6 \mathrm{~cm}^{-1}$.

Propionates of 12 and 13: $\quad$ previously reported ${ }^{12}$

Propionate of 14: $\quad{ }^{1} \mathbf{H}$ NMR $\left(300 \mathrm{MHz} \mathrm{CDCl}_{3}\right) \square 7.36-7.24(\mathrm{~m} ; 5 \mathrm{H}), 5.50(\mathrm{~d} ; \mathrm{J}=6.9 \mathrm{~Hz}$; 1H), 2.43-2.30 (m; 2H), 2.11 (octet; J = 6.9 Hz; 1H), 1.15 (t; J = 7.6 Hz; 3H), 0.98 (d; J = 6.9 Hz; $3 \mathrm{H}), 0.82(\mathrm{~d} ; \mathrm{J}=6.9 \mathrm{~Hz} ; 3 \mathrm{H}) ;{ }^{13} \mathbf{C}$ NMR $\left(75 \mathrm{MHz} \mathrm{CDCl}_{3}\right) \square 173.6,139.9$, 128.1, 127.6, 126.9, 80.6, 33.5, 27.8, 18.7, 18.4, 9.1; MS: HR-EI calculated for $\mathrm{C}_{13} \mathrm{H}_{18} \mathrm{O}_{2}\left(\mathrm{M}^{+}\right) \mathrm{m} / \mathrm{z}$ : 206.1307, measured m/z: 206.1298; IR (KBr) 2964.4, 1738.5, $1186.3 \mathrm{~cm}^{-1}$.

Propionate of 15: $\quad{ }^{1} \mathbf{H}$ NMR $\left(300 \mathrm{MHz} \mathrm{CDCl}_{3}\right) \square 7.3-7.2(\mathrm{~m} ; 5 \mathrm{H}), 5.49(\mathrm{~s} ; 1 \mathrm{H}), 2.37$ (apparent qd; $\left.\mathrm{J}_{1}=7.5 \mathrm{~Hz}, \mathrm{~J}_{2}=3.0 \mathrm{~Hz} ; 2 \mathrm{H}\right), 1.14(\mathrm{t} ; \mathrm{J}=7.5 \mathrm{~Hz} ; 3 \mathrm{H}), 0.92(\mathrm{~s} ; 9 \mathrm{H}) ;{ }^{13} \mathbf{C} \mathbf{N M R}(75$ $\left.\mathrm{MHz}, \mathrm{CDCl}_{3}\right) \square$ 173.4, 138.6, 127.7, 127.5, 127.4, 82.5, 35.0, 27.9, 26.0, 9.2; MS: HR-EI calculated for $\mathrm{C}_{14} \mathrm{H}_{20} \mathrm{O}_{2}\left(\mathrm{M}^{+}\right) \mathrm{m} / \mathrm{z}$ : 220.1463, measured m/z: 220.1455; IR (KBr) 2972.0, 1738.6, $1176.3 \mathrm{~cm}^{-1}$.

Propionate of 16: $\quad{ }^{1} \mathbf{H}$ NMR $\left(300 \mathrm{MHz}, \mathrm{CDCl}_{3}\right) \square 7.87-7.83(\mathrm{~m} ; 4 \mathrm{H}), 7.54-7.51(\mathrm{~m} ; 3 \mathrm{H}), 6.10$ $(\mathrm{q} ; \mathrm{J}=6.6 \mathrm{~Hz} ; 1 \mathrm{H}), 2.41$ (apparent qd; $\left.\mathrm{J}_{1}=7.7 \mathrm{~Hz}, \mathrm{~J}_{2}=1.9 \mathrm{~Hz} ; 2 \mathrm{H}\right), 1.65(\mathrm{~d} ; \mathrm{J}=6.6 \mathrm{H} ; 3 \mathrm{H}), 1.18$ $(\mathrm{t} ; \mathrm{J}=7.7 \mathrm{~Hz} ; 3 \mathrm{H}) ;{ }^{13} \mathbf{C} \mathbf{N M R}\left(75 \mathrm{MHz} \mathrm{CDCl}_{3}\right) \square 173.7,139.1,133.1,132.9,128.3,128.0$, 127.6, 126.1, 124.9, 124.0, 72.1, 27.8, 22.2, 9.1; MS: HR-EI calculated for $\mathrm{C}_{15} \mathrm{H}_{16} \mathrm{O}_{2}\left(\mathrm{M}^{+}\right) \mathrm{m} / \mathrm{z}$ : 228.1150, measured m/z: 228.1154; IR (KBr) 2980.2, 1732.0, $1188.6 \mathrm{~cm}^{-1}$.

Propionate of 17: $\quad{ }^{1} \mathbf{H}$ NMR $\left(300 \mathrm{MHz} \mathrm{CDCl}_{3}\right) \square 8.12(\mathrm{~d} ; \mathrm{J}=8.0 \mathrm{~Hz} ; 1 \mathrm{H}), 7.88-7.80$ (m; $2 \mathrm{H}), 7.63-7.46(\mathrm{~m} ; 4 \mathrm{H}), 6.69(\mathrm{q} ; \mathrm{J}=6.6 \mathrm{H} ; 1 \mathrm{H}), 2.42$ (apparent qd; $\left.\mathrm{J}_{1}=7.7 \mathrm{~Hz}, \mathrm{~J}_{2}=2.2 \mathrm{~Hz} ; 2 \mathrm{H}\right)$, $1.72(\mathrm{~d} ; \mathrm{J}=6.6 \mathrm{~Hz} ; 3 \mathrm{H}), 1.18(\mathrm{t} ; \mathrm{J}=7.7 \mathrm{~Hz} ; 3 \mathrm{H}) ;{ }^{13} \mathbf{C} \mathbf{N M R}\left(75 \mathrm{MHz}, \mathrm{CDCl}_{3}\right) \square$ 173.7, 137.6, 133.8, 130.2, 128.9, 128.3, 126.2, 125.6, 125.3, 123.2, 123.1, 69.2, 27.9, 21.7, 9.1; MS: HR-EI calculated for $\mathrm{C}_{15} \mathrm{H}_{16} \mathrm{O}_{2}\left(\mathrm{M}^{+}\right) \mathrm{m} / \mathrm{z}$ : 228.1150, measured m/z: 228.1160; IR (KBr) 2980.9, 1735.5, $1186.1 \mathrm{~cm}^{-1}$. 
Propionate of 18: $\quad{ }^{1} \mathbf{H}$ NMR $\left(300 \mathrm{MHz}, \mathrm{CDCl}_{3}\right) \square 5.68$ (apparent doublet; J = $\left.0.8 \mathrm{~Hz} ; 1 \mathrm{H}\right)$, $5.23(\mathrm{q} ; \mathrm{J}=6.4 \mathrm{~Hz} ; 1 \mathrm{H}), 2.30(\mathrm{q} ; \mathrm{J}=7.4 \mathrm{~Hz} ; 2 \mathrm{H}), 2.0-1.95(\mathrm{~m} ; 4 \mathrm{H}), 1.6-1.5(\mathrm{~m} ; 4 \mathrm{H}), 1.27(\mathrm{~d} ; \mathrm{J}=$ $6.4 \mathrm{~Hz} ; 3 \mathrm{H}), 1.12(\mathrm{t} ; \mathrm{J}=7.4 \mathrm{~Hz} ; 3 \mathrm{H}) ;{ }^{13} \mathbf{C} \mathbf{~ N M R}\left(75 \mathrm{MHz}, \mathrm{CDCl}_{3}\right) \square$ 173.8, 137.2, 123.4, 73.8, 27.9, 24.8, 24.1, 22.4, 22.3, 18.8, 9.1; MS: HR-EI calculated for $\mathrm{C}_{11} \mathrm{H}_{18} \mathrm{O}_{2}\left(\mathrm{M}^{+}\right) \mathrm{m} / \mathrm{z}$ : 182.1307, measured m/z: 182.1306; IR $\left(\mathrm{KBr}, \mathrm{cm}^{-1}\right)$ 2932.0, 1735.7, 1192.4 .

Benzoate of 18: ${ }^{1} \mathbf{H}$ NMR $\left(300 \mathrm{MHz}, \mathrm{CDCl}_{3}\right) \square 8.05(\mathrm{~d} ; \mathrm{J}=7.4 \mathrm{~Hz} ; 2 \mathrm{H}), 7.55(\mathrm{t} ; \mathrm{J}=7.4 \mathrm{~Hz}$; $1 \mathrm{H}), 7.43(\mathrm{t} ; \mathrm{J}=7.4 \mathrm{~Hz} ; 2 \mathrm{H}), 5.79$ (apparent doublet; $\mathrm{J}=0.8 \mathrm{~Hz} ; 1 \mathrm{H}), 5.50(\mathrm{q} ; \mathrm{J}=6.4 \mathrm{~Hz} ; 1 \mathrm{H})$, 2.1-2.0 (m; 4H), 1.75-1.55 (m; 4H), $1.43(\mathrm{~d} ; \mathrm{J}=6.4 \mathrm{~Hz} ; 3 \mathrm{H}) ;{ }^{13} \mathbf{C}$ NMR $\left(75 \mathrm{MHz}, \mathrm{CDCl}_{3}\right) \square$ 165.8, 137.1, 132.7, 130.9, 129.5, 128.2, 123.7, 74.8, 24.8, 24.1, 22.5, 22.3, 19.0; MS: HR-EI calculated for $\mathrm{C}_{15} \mathrm{H}_{18} \mathrm{O}_{2}\left(\mathrm{M}^{+}\right) \mathrm{m} / \mathrm{z}: 230.1307$, measured m/z: 230.1314; IR $\left(\mathrm{KBr}, \mathrm{cm}^{-1}\right)$ 2929.0, $1716.8,1271.8$.

HPLC separation and properties of alcohol substrates.

\begin{tabular}{|c|c|c|c|c|c|}
\hline \multirow[b]{2}{*}{ Substrate } & \multirow{2}{*}{$\begin{array}{c}\text { HPLC } \\
\text { conditions }^{\mathrm{a}}\end{array}$} & \multicolumn{2}{|c|}{ Retention time $(\min )^{\mathrm{a}}$} & \multicolumn{2}{|c|}{ Unreacted alcohol $^{\mathrm{d}}$} \\
\hline & & (R)-ent & $(\mathrm{S})$-ent & $\begin{array}{l}\text { Sign of } \\
\text { rotation }\end{array}$ & Ref. \\
\hline & $\begin{array}{c}3 \% \\
\text { IPA/hexane }\end{array}$ & 21.2 & 35.3 & $(-)$ & 13 \\
\hline & $\begin{array}{c}5 \% \\
\text { IPA/hexane }\end{array}$ & 14.5 & 23.1 & $(+)$ & 14 \\
\hline & $\begin{array}{c}5 \% \\
\text { IPA/hexane }\end{array}$ & 12.9 & 14.6 & $(+)$ & 15 \\
\hline & $\begin{array}{c}1 \% \\
\text { IPA/hexane }\end{array}$ & 32.5 & 37.3 & $(+)$ & 16 \\
\hline & $\begin{array}{c}18 \% \\
\text { IPA/hexane }\end{array}$ & 11.1 & 19.7 & $(-)$ & 17 \\
\hline & $\begin{array}{c}0.5 \% \\
\text { IPA/hexane }\end{array}$ & $13.9^{b}$ & $19.8^{b}$ & $(-)$ & 17 \\
\hline & $\begin{array}{c}2 \% \\
\text { IPA/hexane }\end{array}$ & 20.7 & 26.2 & $(-)$ & 18 \\
\hline & $\begin{array}{c}2.5 \% \\
\text { IPA/hexane }\end{array}$ & 23.4 & 26.0 & $(-)$ & 18 \\
\hline $14^{\mathrm{Me}}$ & $\begin{array}{c}1 \% \\
\text { IPA/hexane }\end{array}$ & 41.7 & 34.6 & $(-)$ & 18 \\
\hline
\end{tabular}




\begin{tabular}{|l|l|l|l|l|l|}
\hline & $\begin{array}{c}2 \% \\
\text { IPA/hexane }\end{array}$ & 21.1 & 13.7 & $(-)$ & 19 \\
\hline & $\begin{array}{c}10 \% \\
\text { IPA/hexane }\end{array}$ & 21.1 & 13.1 & $(-)$ & 18 \\
\hline & $100 \%$ hexane & $27.2^{\mathrm{b}}$ & $32.5^{\mathrm{b}}$ & $(-)$ & 20 \\
\hline
\end{tabular}

a) Unless specified otherwise, HPLC separation of enantiomers was achieved using free alcohols on CHIRALCEL OD-H analytical column $(4.6 \times 250 \mathrm{~mm}$, Chiral Technologies, Inc.) with isopropanol/hexane eluent of the concentration indicated;

$\left.{ }^{b}\right)$ HPLC separation of enantiomers was achieved using the propionate esters of the alcohols on CHIRALPAK AD analytical column (4.6x250 mm, Chiral Technologies, Inc.)

c) HPLC separation of enantiomers was achieved using the benzoate ester of the alcohol on CHIRALPAK AD analytical column (4.6x250 mm, Chiral Technologies, Inc.)

$\left.{ }^{d}\right)$ Absolute configuration was confirmed to be $\mathrm{S}$ in all cases

\section{Kinetic resolution data}

\begin{tabular}{|c|c|c|c|c|c|c|c|c|c|c|}
\hline Entry & Substrate & Catalyst & $\mathbf{t}(\mathbf{h})$ & $\#$ & $\begin{array}{c}\mathbf{e e}_{\mathrm{E}} \\
\%\end{array}$ & $\begin{array}{c}\mathbf{e e}_{\mathrm{A}} \\
\%\end{array}$ & $\begin{array}{c}\mathbf{C}_{\text {HPLC }} \\
\%\end{array}$ & $\mathbf{S}$ & $\begin{array}{c}\mathrm{C}_{\mathrm{AVG}} \\
\%\end{array}$ & $\mathbf{S}_{\mathrm{AVG}}$ \\
\hline \multirow{2}{*}{1} & & \multirow{2}{*}{$\mathrm{CF}_{3} \mathrm{PIP}$} & \multirow{2}{*}{8} & 1 & 79.1 & 11.8 & 13.0 & 9.6 & \multirow{2}{*}{14} & \multirow{2}{*}{11} \\
\hline & & & & 2 & 82.2 & 13.6 & 14.2 & 11.7 & & \\
\hline \multirow{2}{*}{2} & & \multirow{2}{*}{ Cl-PIQ } & \multirow{2}{*}{8} & 1 & 86.3 & 66.1 & 43.4 & 26.9 & \multirow{2}{*}{44} & \multirow{2}{*}{27} \\
\hline & & & & 2 & 85.7 & 68.7 & 44.5 & 26.7 & & \\
\hline \multirow{2}{*}{3} & & \multirow{2}{*}{$\mathrm{CF}_{3} \mathrm{PIP}$} & \multirow{2}{*}{8} & 1 & 86.9 & 39.2 & 31.1 & 21.0 & \multirow{2}{*}{30} & \multirow{2}{*}{21} \\
\hline & & & & 2 & 86.9 & 36.4 & 29.5 & 20.4 & & \\
\hline \multirow{2}{*}{4} & & \multirow{2}{*}{ Cl-PIQ } & \multirow{2}{*}{8} & 1 & 75.9 & 90.5 & 54.4 & 22.3 & \multirow{2}{*}{53} & \multirow{2}{*}{24} \\
\hline & & & & 2 & 81.3 & 84.8 & 51.1 & 25.9 & & \\
\hline \multirow{2}{*}{5} & & \multirow{2}{*}{$\mathrm{CF}_{3} \mathrm{PIP}$} & \multirow[t]{2}{*}{8} & 1 & 75.2 & 12.4 & 14.1 & 8.0 & \multirow{2}{*}{14} & \multirow{2}{*}{9} \\
\hline & & & & 2 & 80.9 & 13.4 & 14.2 & 10.8 & & \\
\hline \multirow{2}{*}{6} & & \multirow{2}{*}{ Cl-PIQ } & \multirow{2}{*}{8} & 1 & 82.1 & 53.5 & 39.4 & 17.3 & \multirow{2}{*}{38} & \multirow{2}{*}{17} \\
\hline & & & & 2 & 82.0 & 45.7 & 35.8 & 15.8 & & \\
\hline \multirow{2}{*}{7} & & \multirow{2}{*}{$\mathrm{CF}_{3} \mathrm{PIP}$} & \multirow[t]{2}{*}{8} & 1 & 84.1 & 8.7 & 9.4 & 12.6 & 0 & 13 \\
\hline & & & & 2 & 84.0 & 8.4 & 9.1 & 12.5 & 9 & 15 \\
\hline 8 & & Cl_PIO & 8 & 1 & 86.6 & 42.3 & 32.8 & 21.1 & 32 & 22 \\
\hline & & CI-PIQ & $\gamma$ & 2 & 88.2 & 39.7 & 31.0 & 23.6 & 32 & 22 \\
\hline 9 & & CF PIP & 8 & 1 & 61.6 & 23.9 & 27.9 & 5.3 & 27 & 6 \\
\hline & & $C \Gamma_{3}$ & & 2 & 71.6 & 24.2 & 25.3 & 7.6 & 21 & 0 \\
\hline 10 & & & 8 & 1 & 75.7 & 96.3 & 56.0 & 28.1 & 5 & 31 \\
\hline 10 & & Q & 8 & 2 & 77.8 & 97.2 & 55.6 & 33.3 & 50 & 31 \\
\hline
\end{tabular}




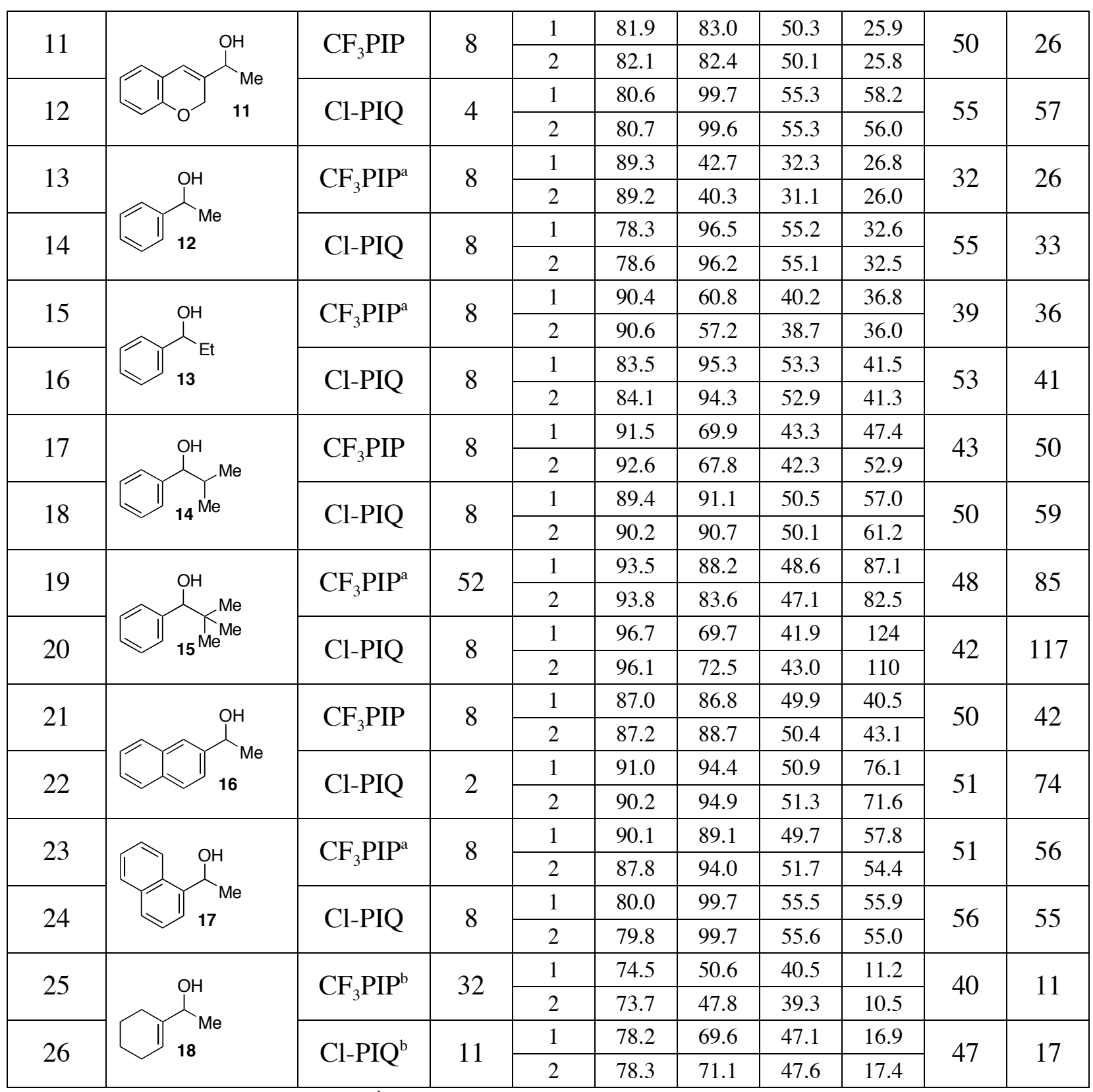

$\left.{ }^{a}\right)$ Data from previously published work. ${ }^{1}$

b) $10 \mathrm{~mol} \%$ of the catalyst was used

\section{ADDitional REFERENCES}

9. $\mathrm{CeCl}_{3}-\mathrm{NaBH}_{4}$ procedure was employed in this case: Gemal, A. L.; Luche, J.-L. J. Am. Chem. Soc. 1981, 103, 5454.

10. (a) Braude, E. A.; Fawcet, J. S. J. Chem. Soc. 1952, 1528; (b) Nechepurenko, I. V.; Shul'ts, E. E.; Tolstikov, G. A. Russ. J. Org. Chem. 2001, 37, 1276.

11. (a) Brion, J.-D.; Le Baut, G.; Ducrey, P.; Sylvie, P.-R.; Cudennec, C.; Genevieve, S. Eur. Patents 0338895 and 0337885, 1989; (b) Alami, M.; Peyrat, J.-F.; Belachmi, L.; Brion, J.-D. Eur. J. Org. Chem. 2001, 4207.

12. Sydnes, L. L.; Sandberg, M. Tetrahedron 1997, 53, 12679. 
13. Fornasier, R.; Reniero, F.; Scrimin, P.; Tonellato, U. J. Org. Chem. 1985, 50, 3209.

14. Ohkuma, T.; Koizumi, M.; Doucet, H.; Pham, T.; Kozawa, M.; Murata, K.; Katayama, E.; Yokozawa, T.; Ikariya, T.; Noyori, R. J. Am. Chem. Soc. 1998, 120, 13529.

15. Fuganti, C.; Grasselli, P.; Spreafico, F.; Zirotti, C.; Casati, P. J. Chem. Res. ( S) 1985, 22.

16. Miller, K. M.; Huang, W.-S.; Jamison, T. F. J. Am. Chem. Soc. 2003, 125, 3442.

17. The absolute configuration was assigned by Mosher ester analysis: Dale, J. A.; Dull, D. L.; Mosher, H. S. J. Org. Chem. 1969, 34, 2543.

18. Aldrich catalog 2005-2006.

19. Clark, D. R.; Mosher, H. S. J. Org. Chem. 1970, 35, 1114.

10. Gao, Y.; Hanson, R. M.; Klunder, J. M.; Ko, S. Y.; Masamune, H.; Sharpless, K. B. J. Am. Chem. Soc. 1987, 109, 5765. 


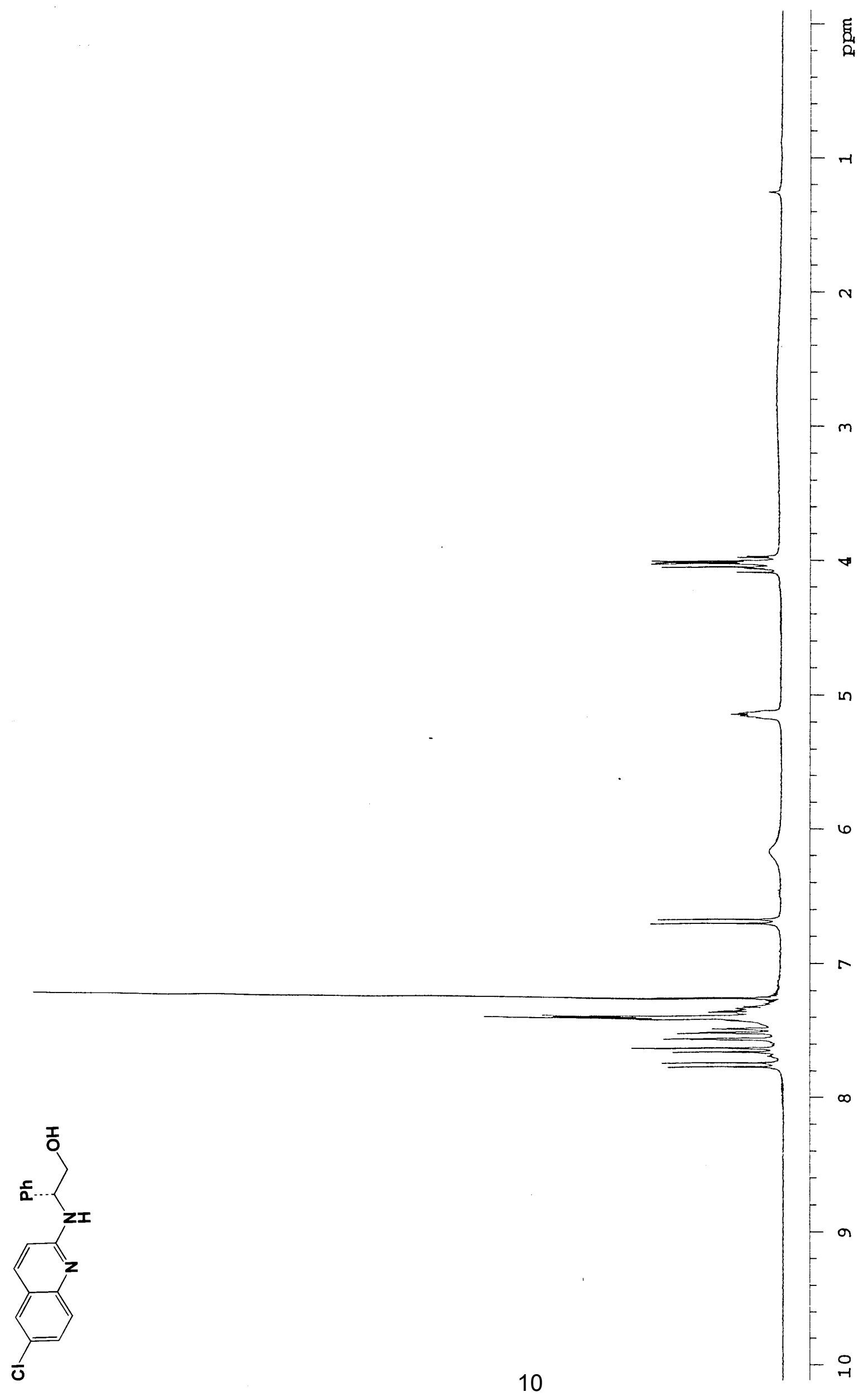




$$
4
$$




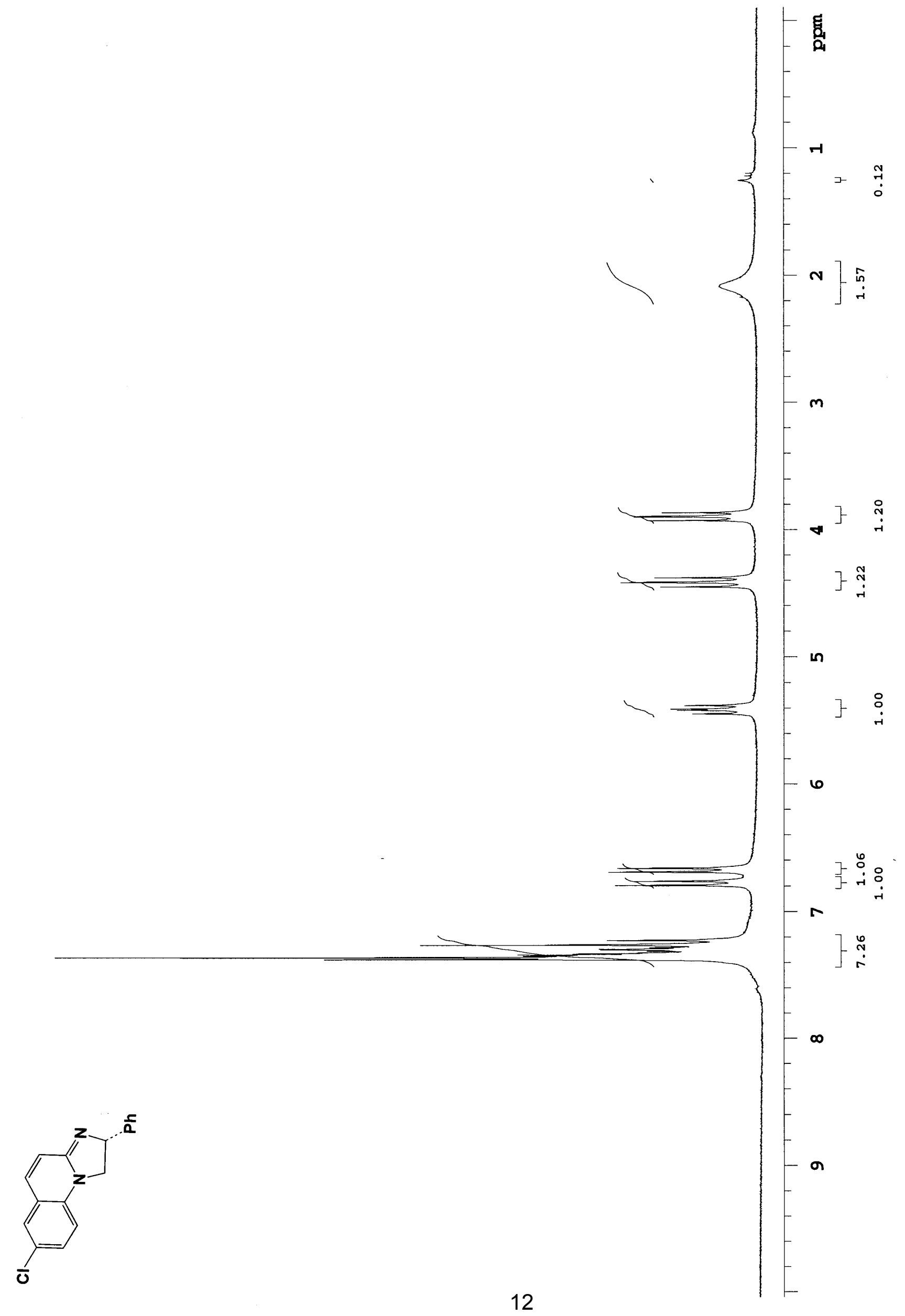




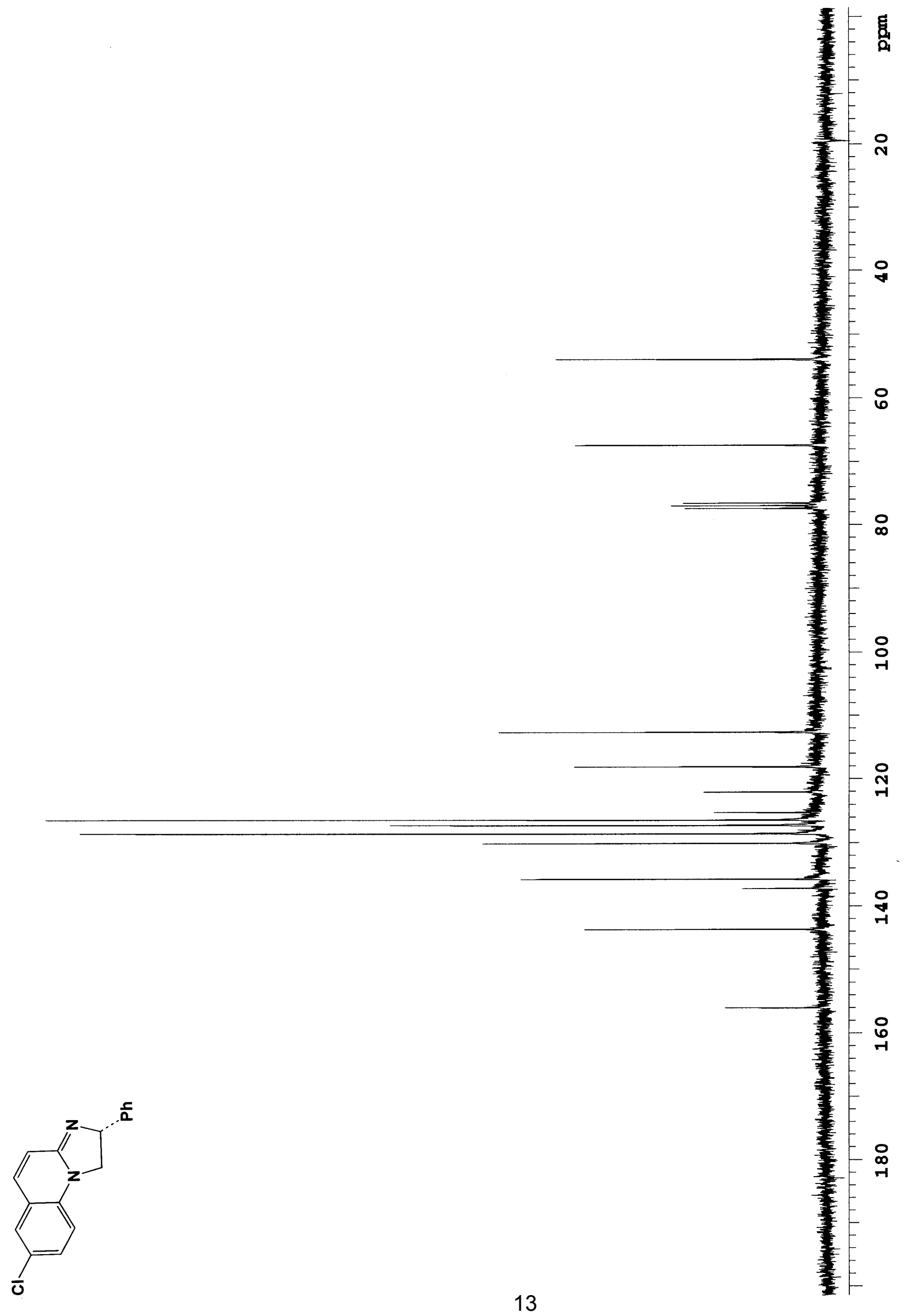




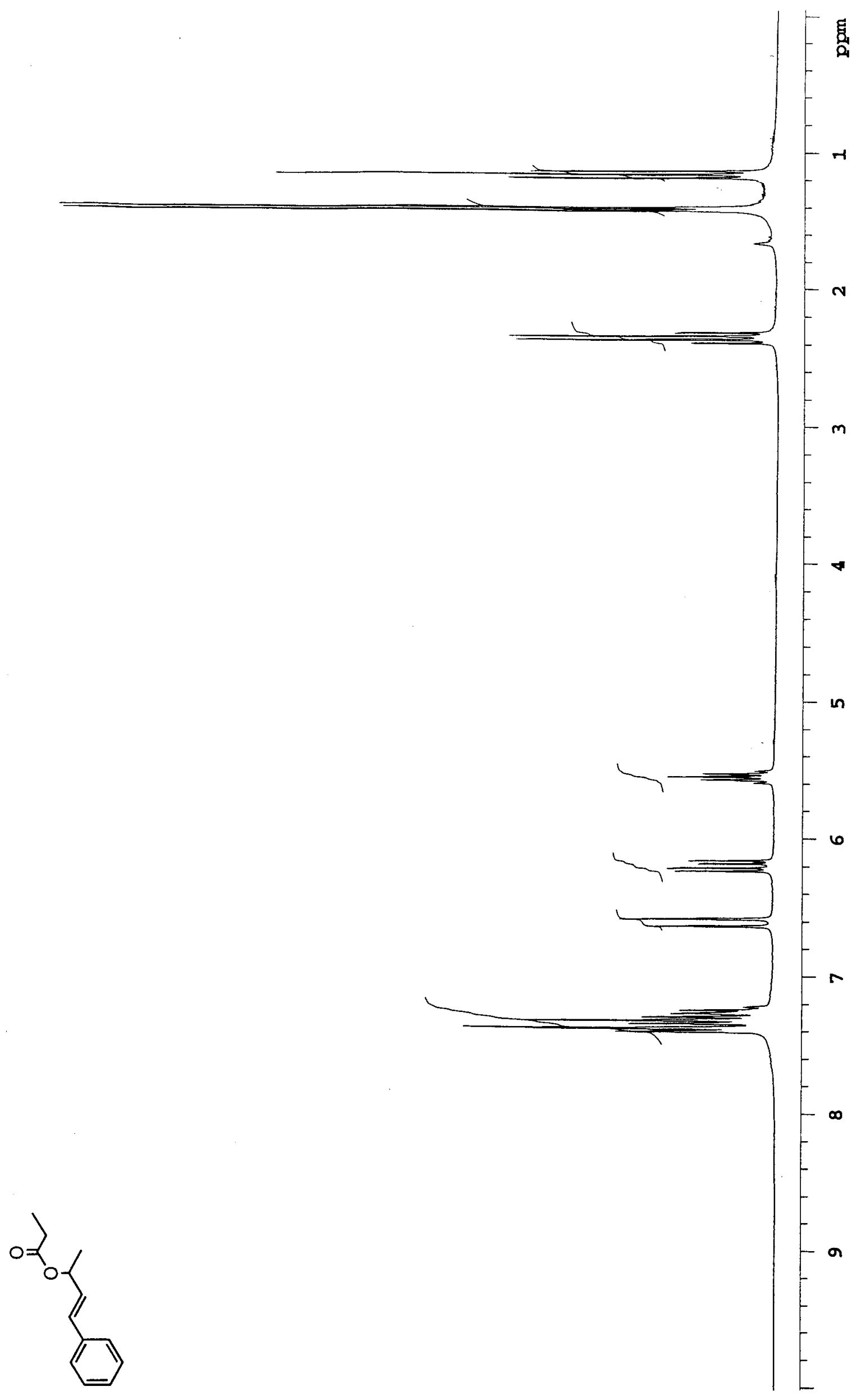




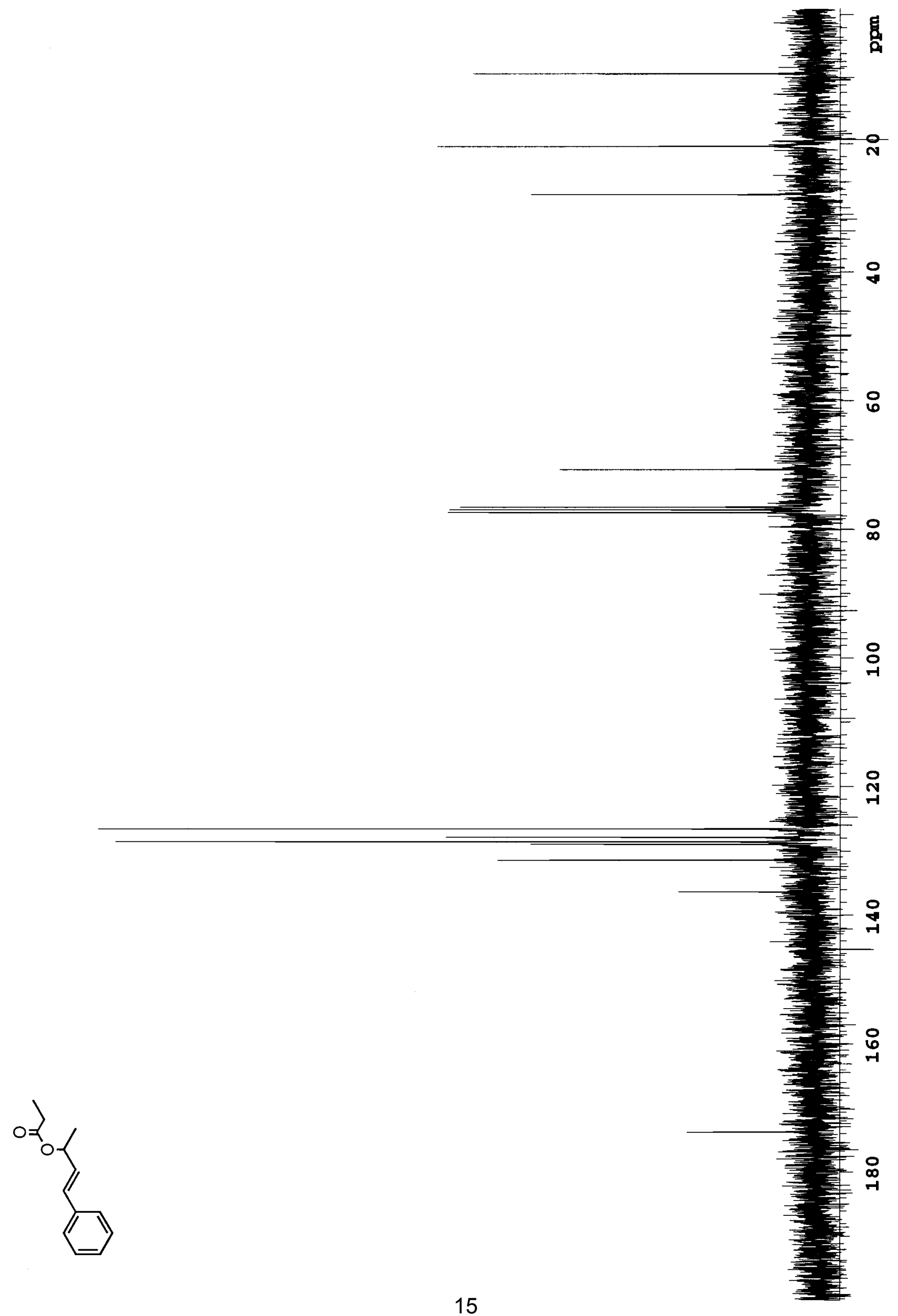




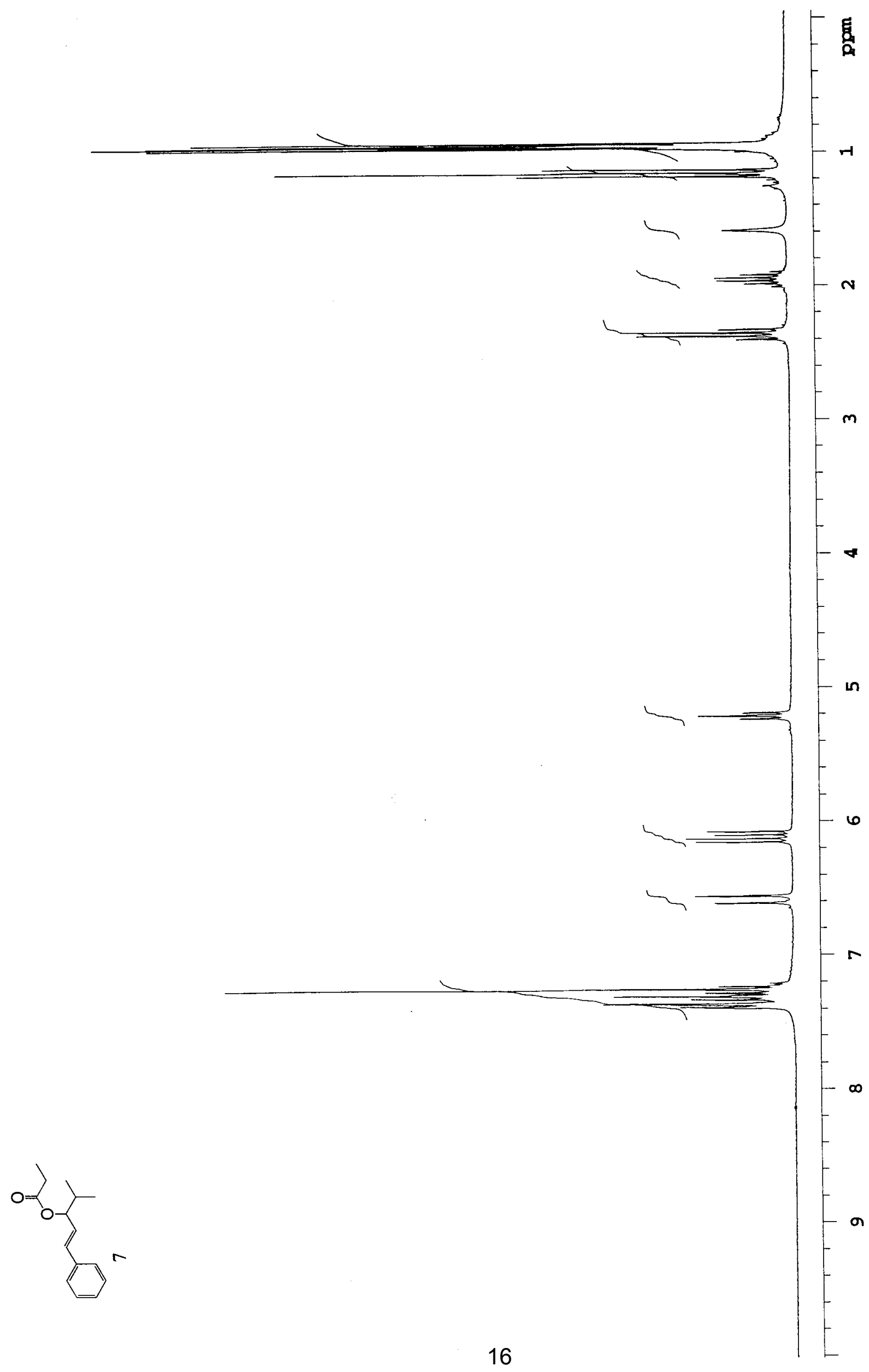




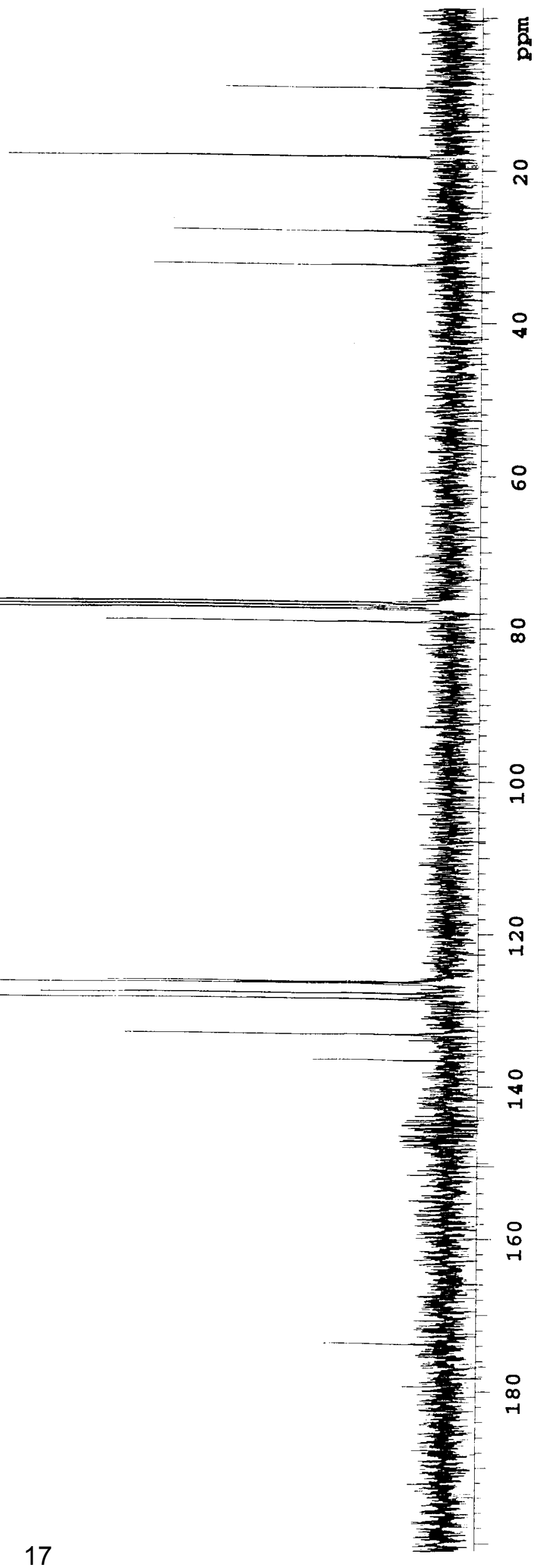




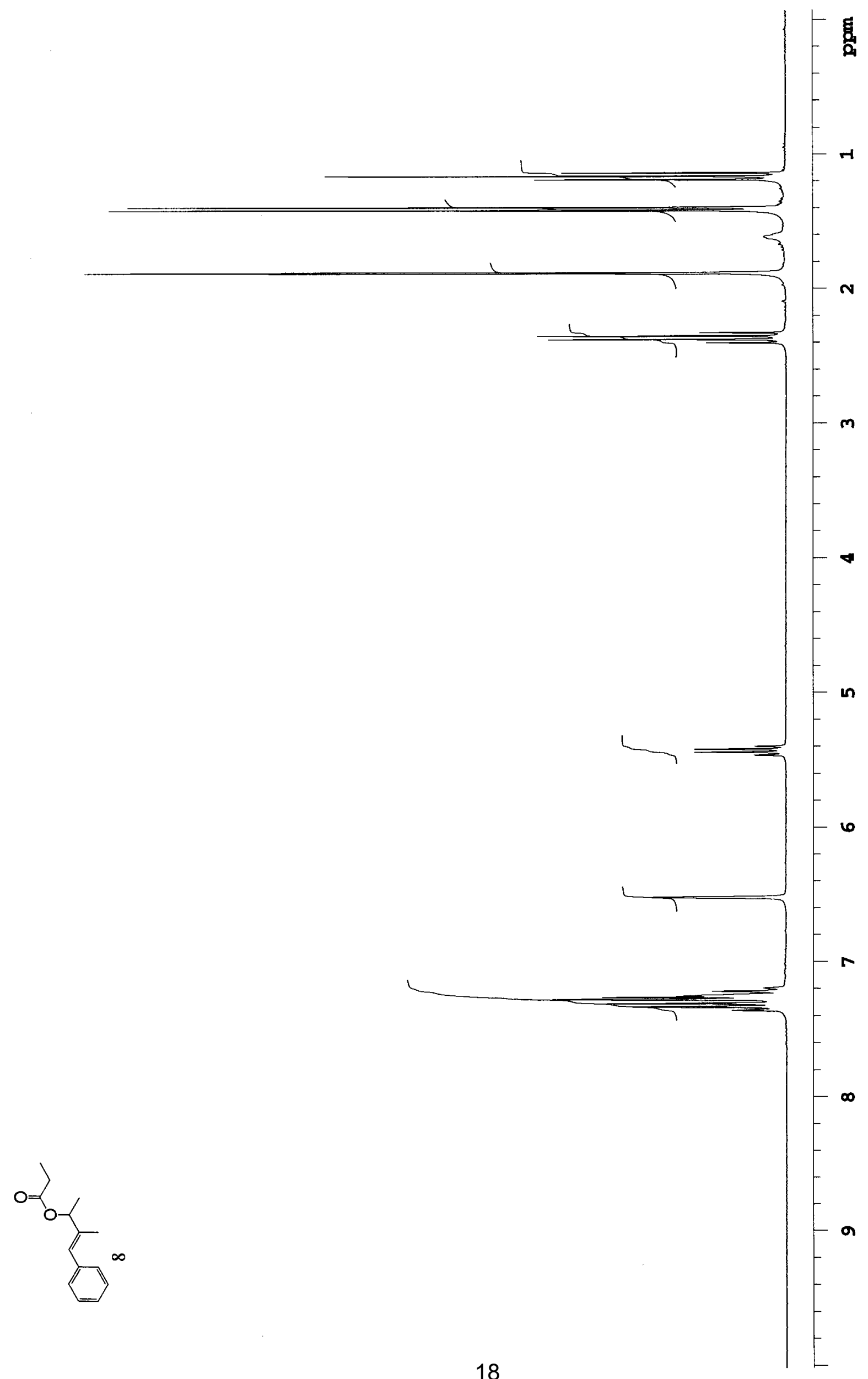




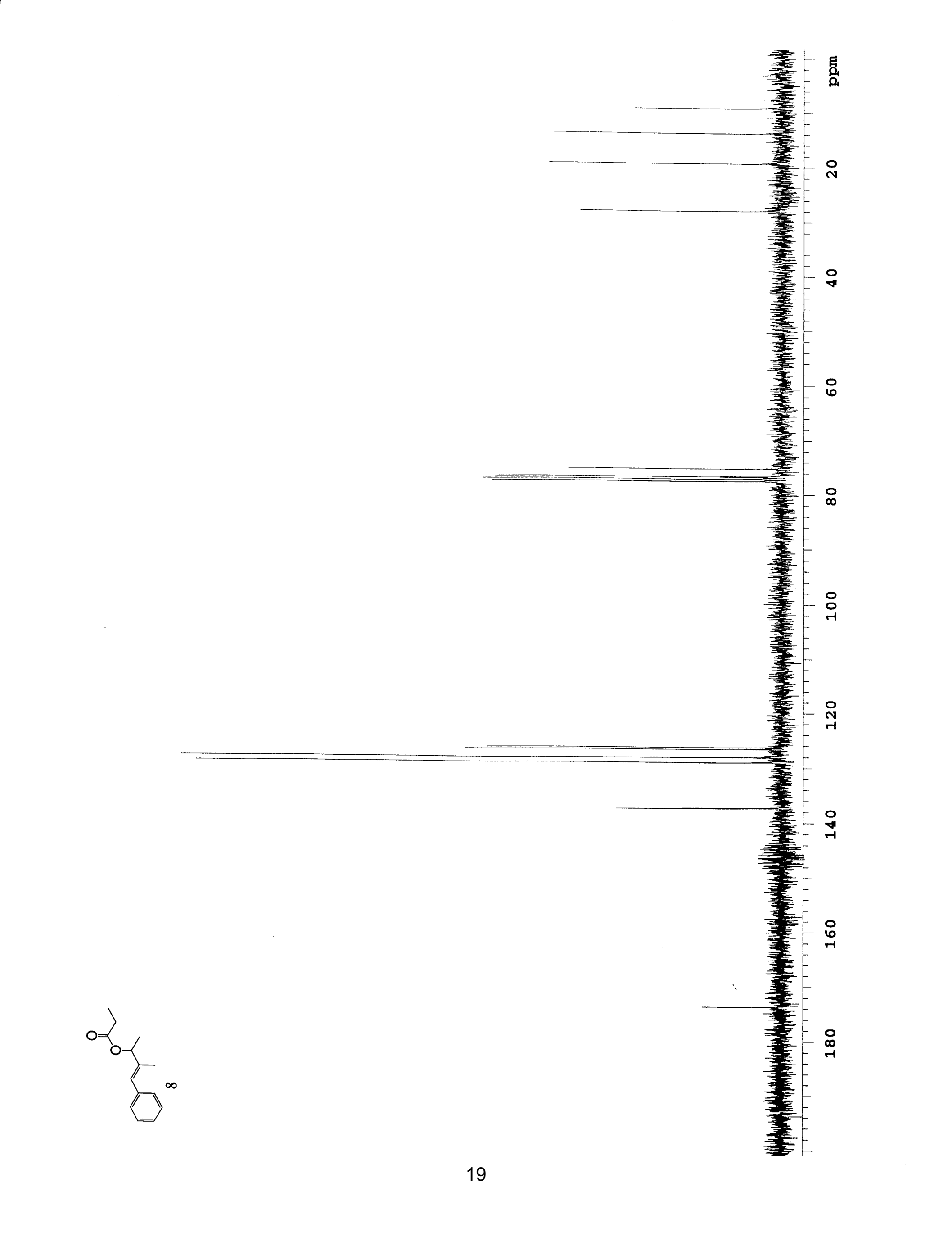




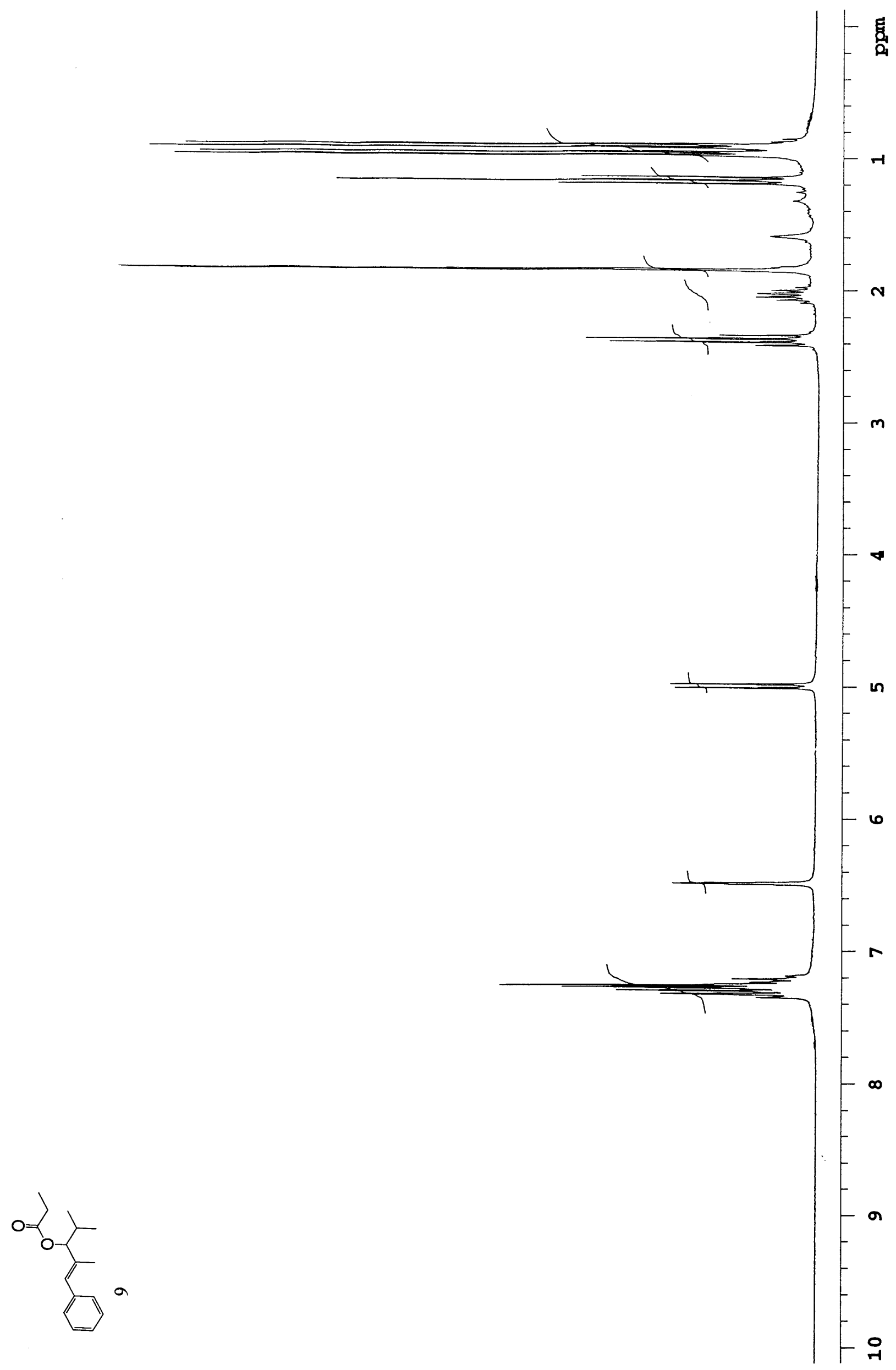




$$
\exists
$$




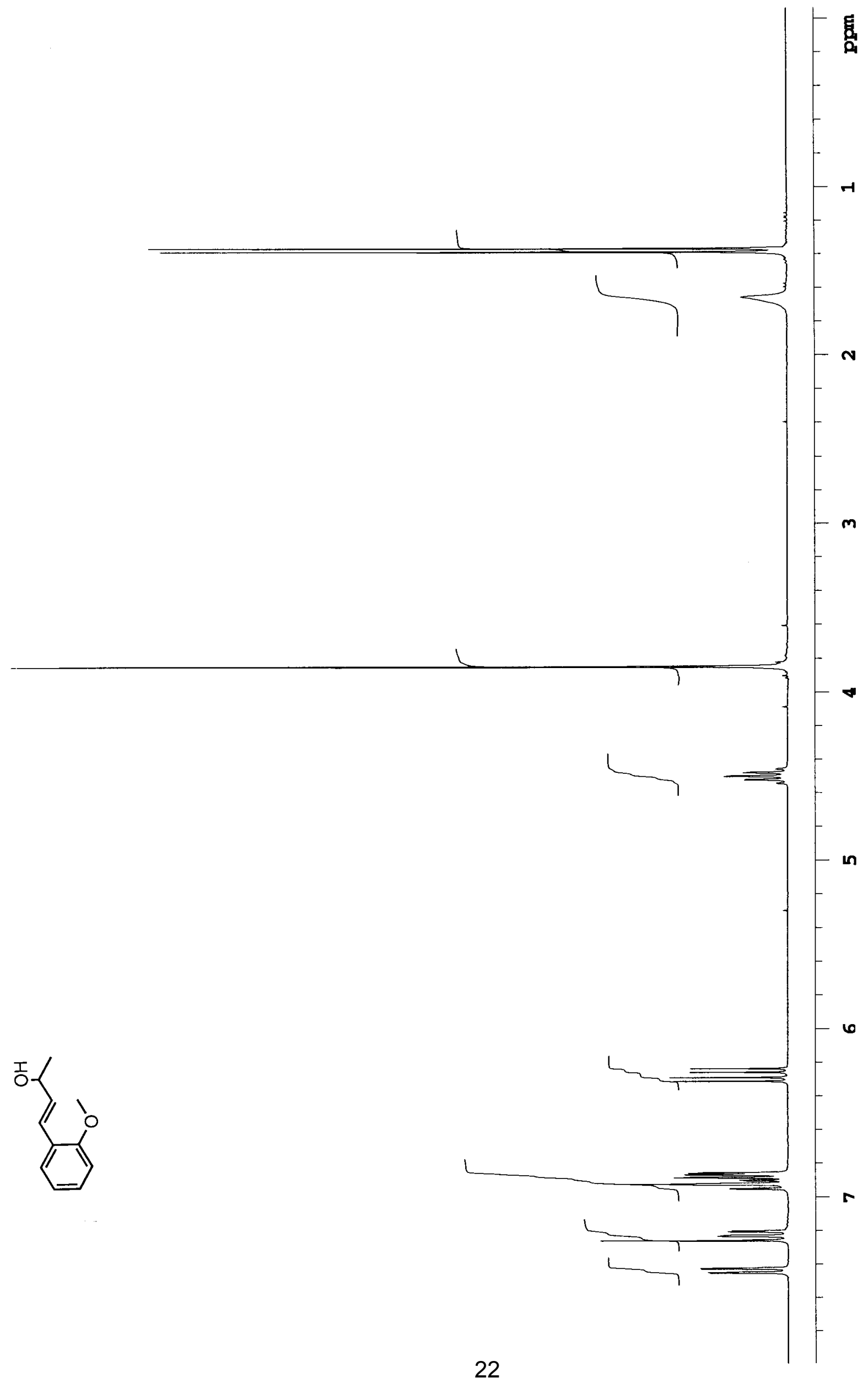




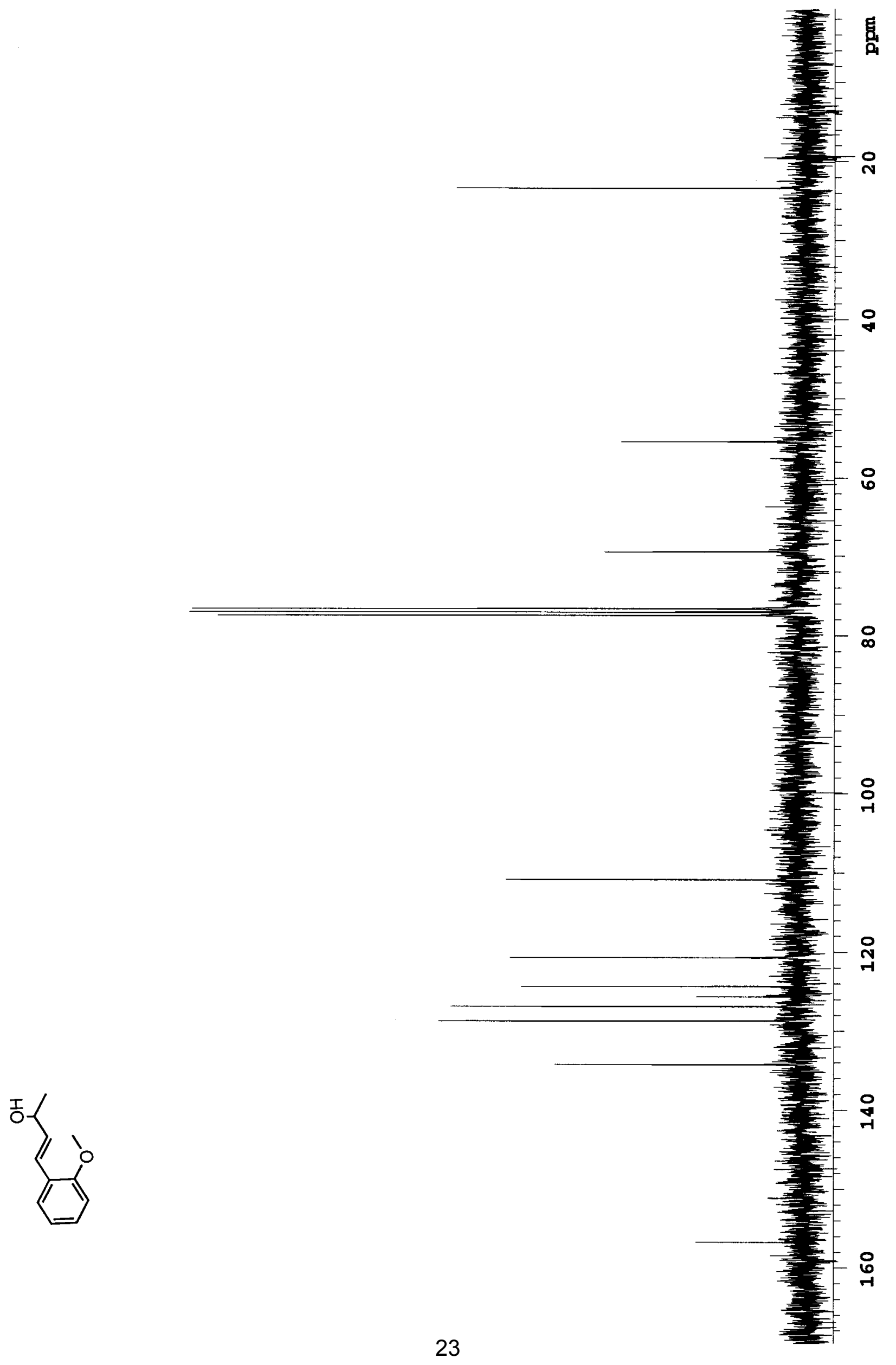




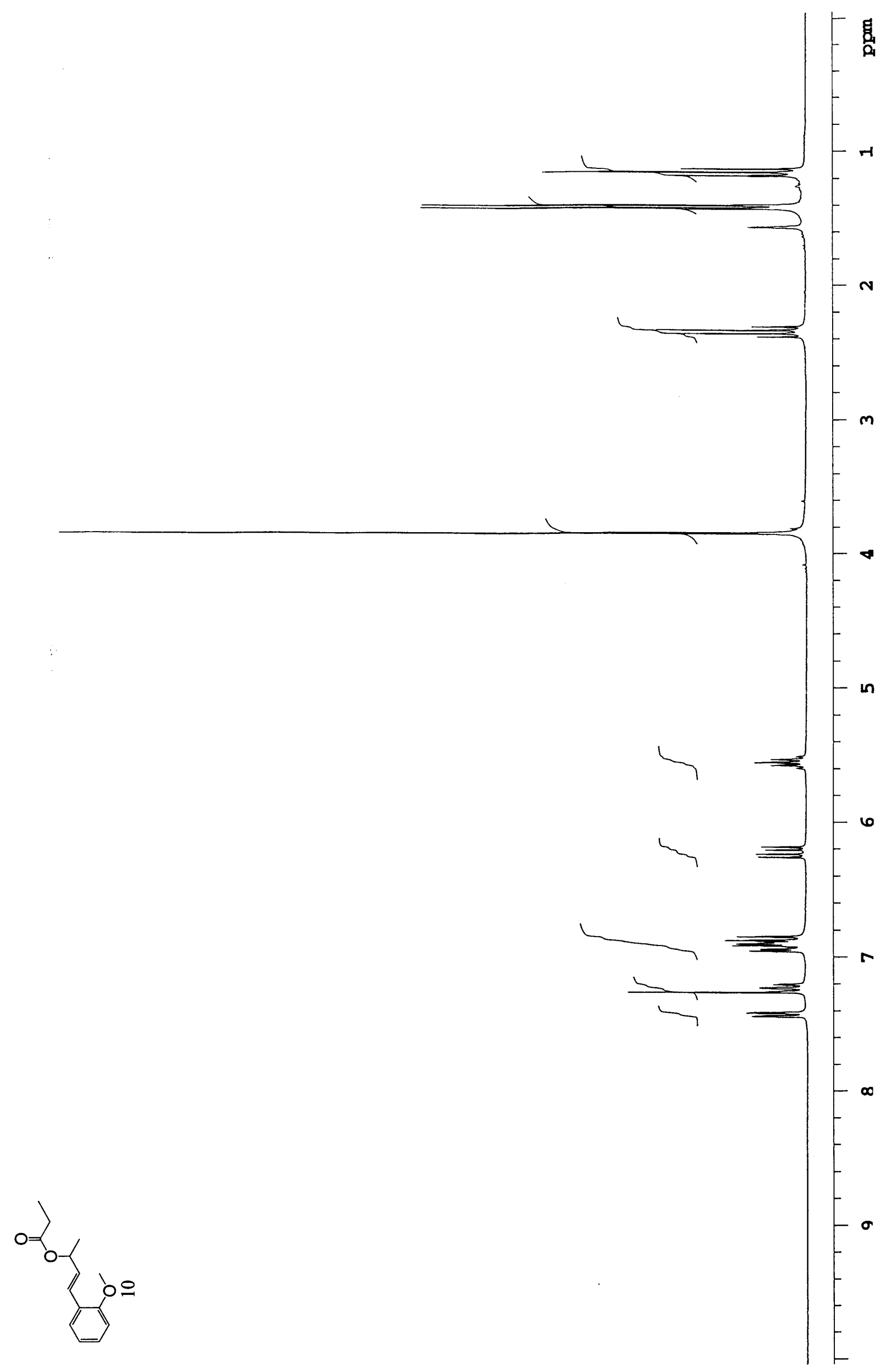




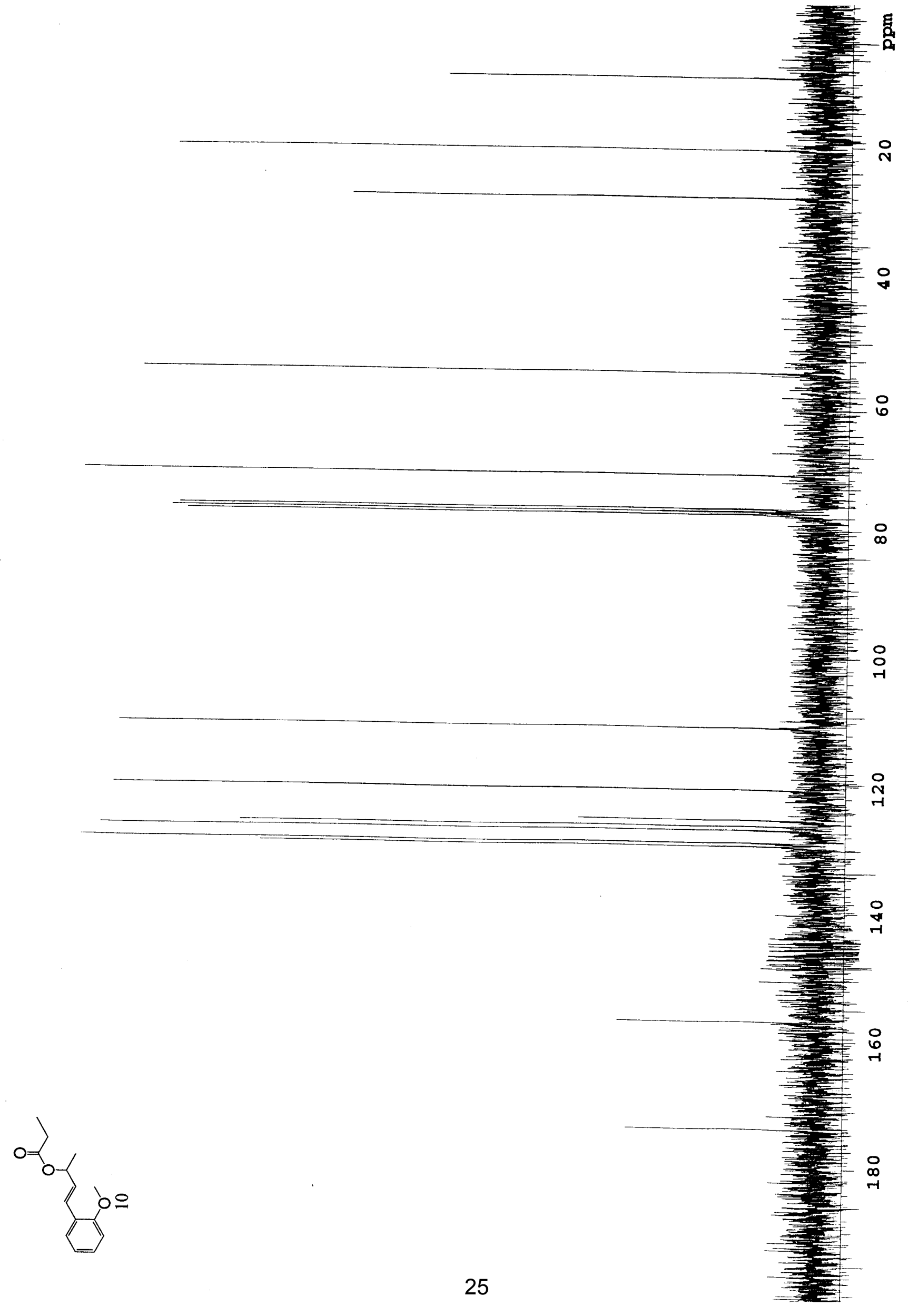




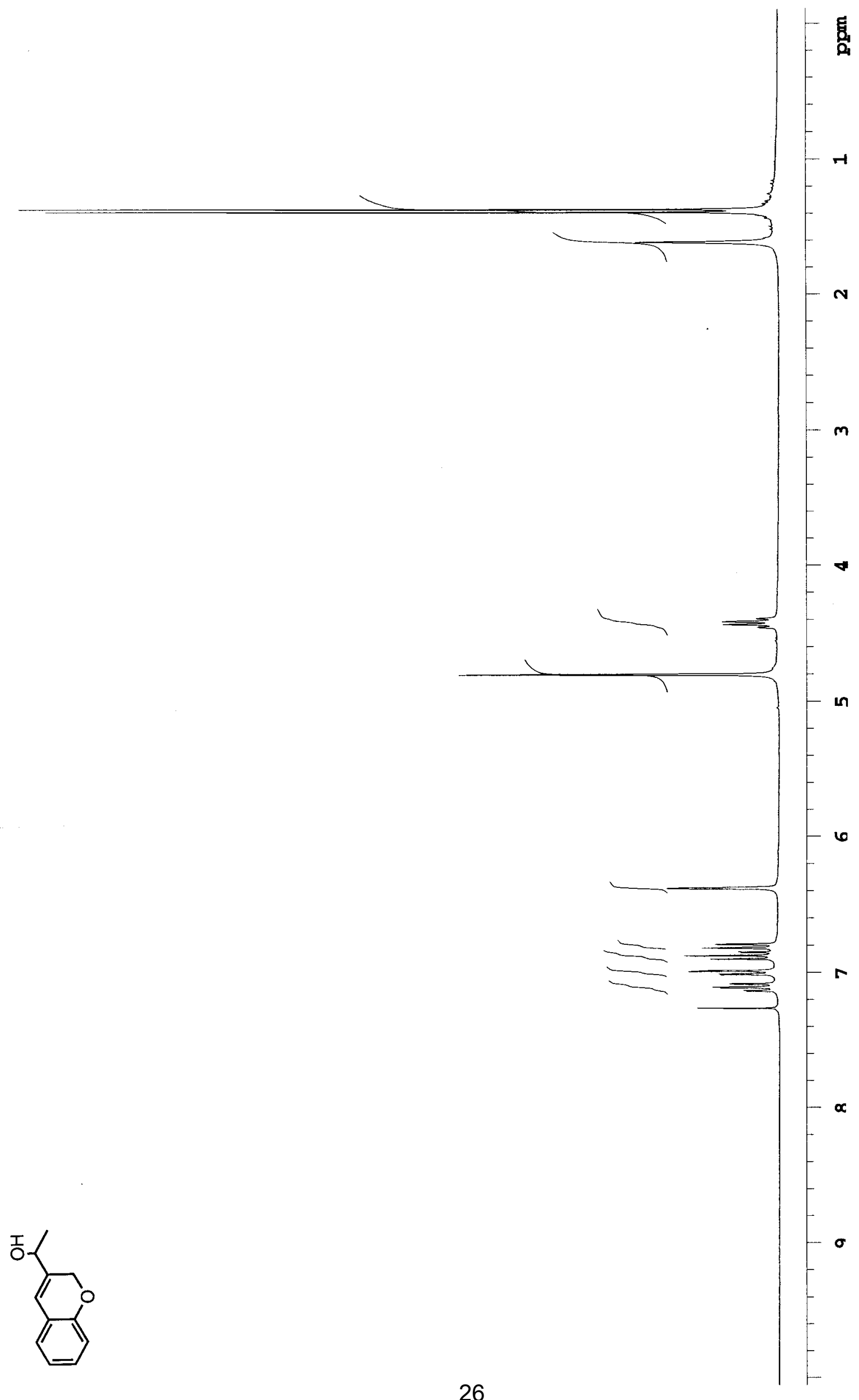




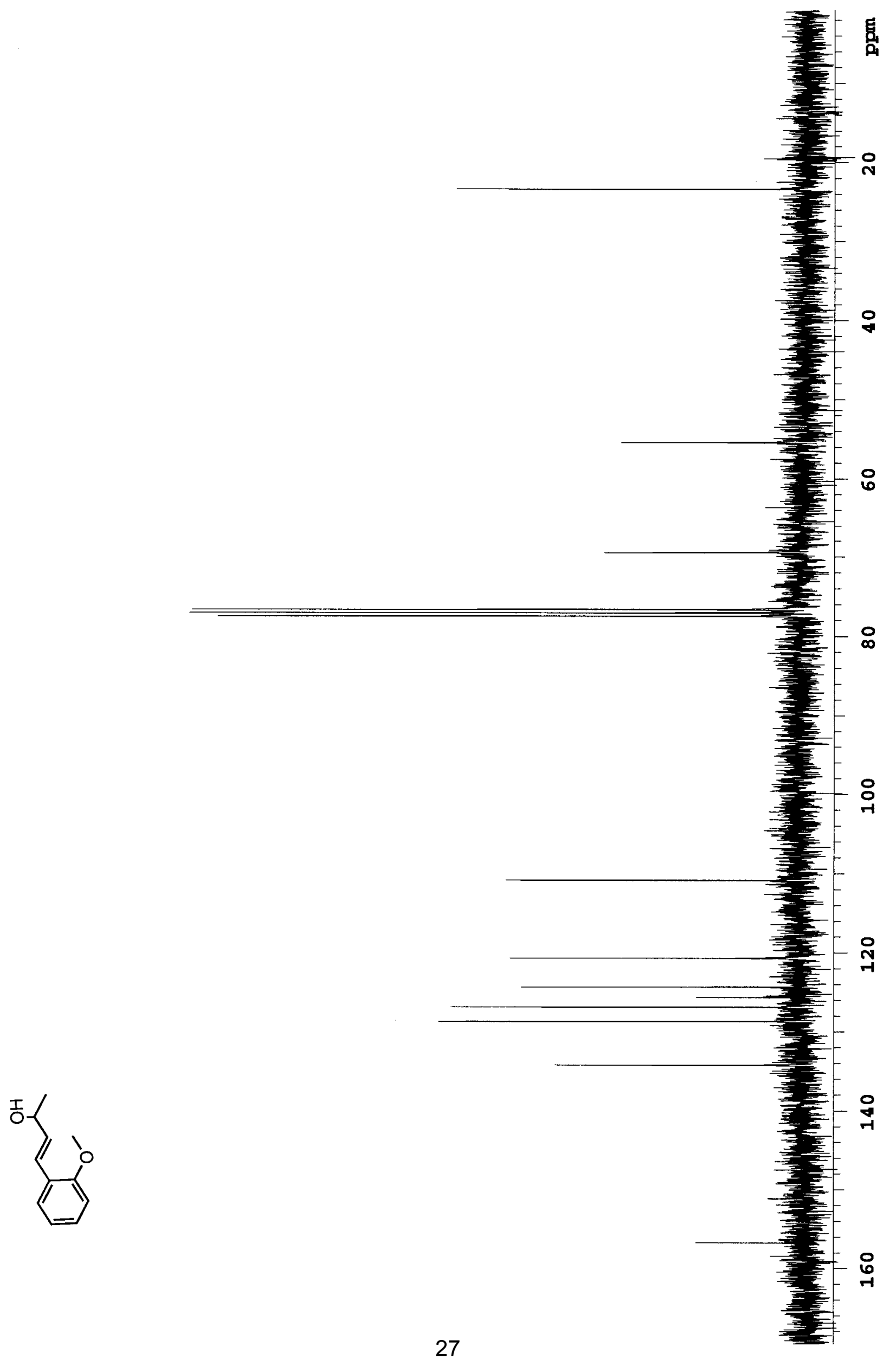




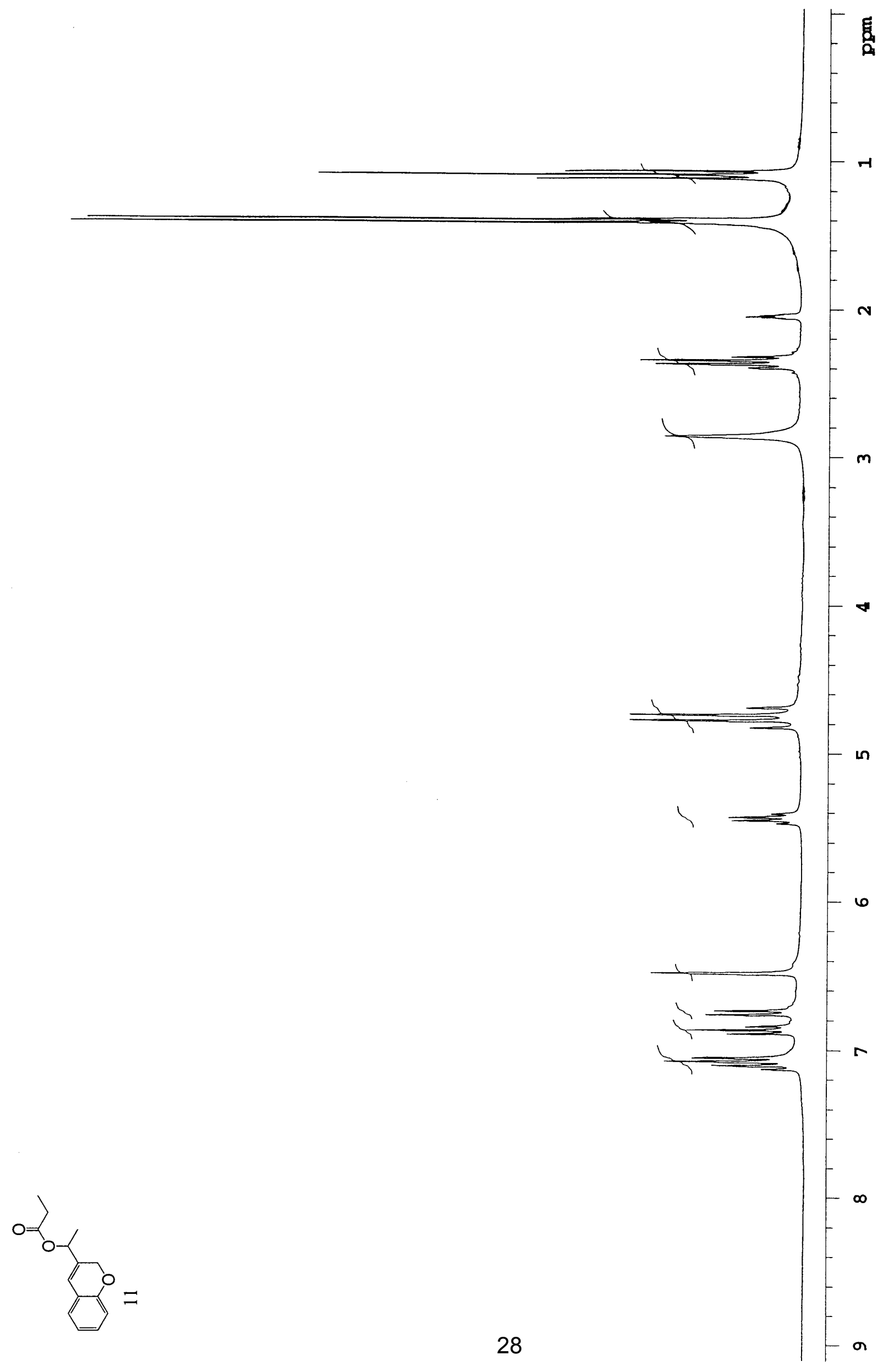




$$
1
$$




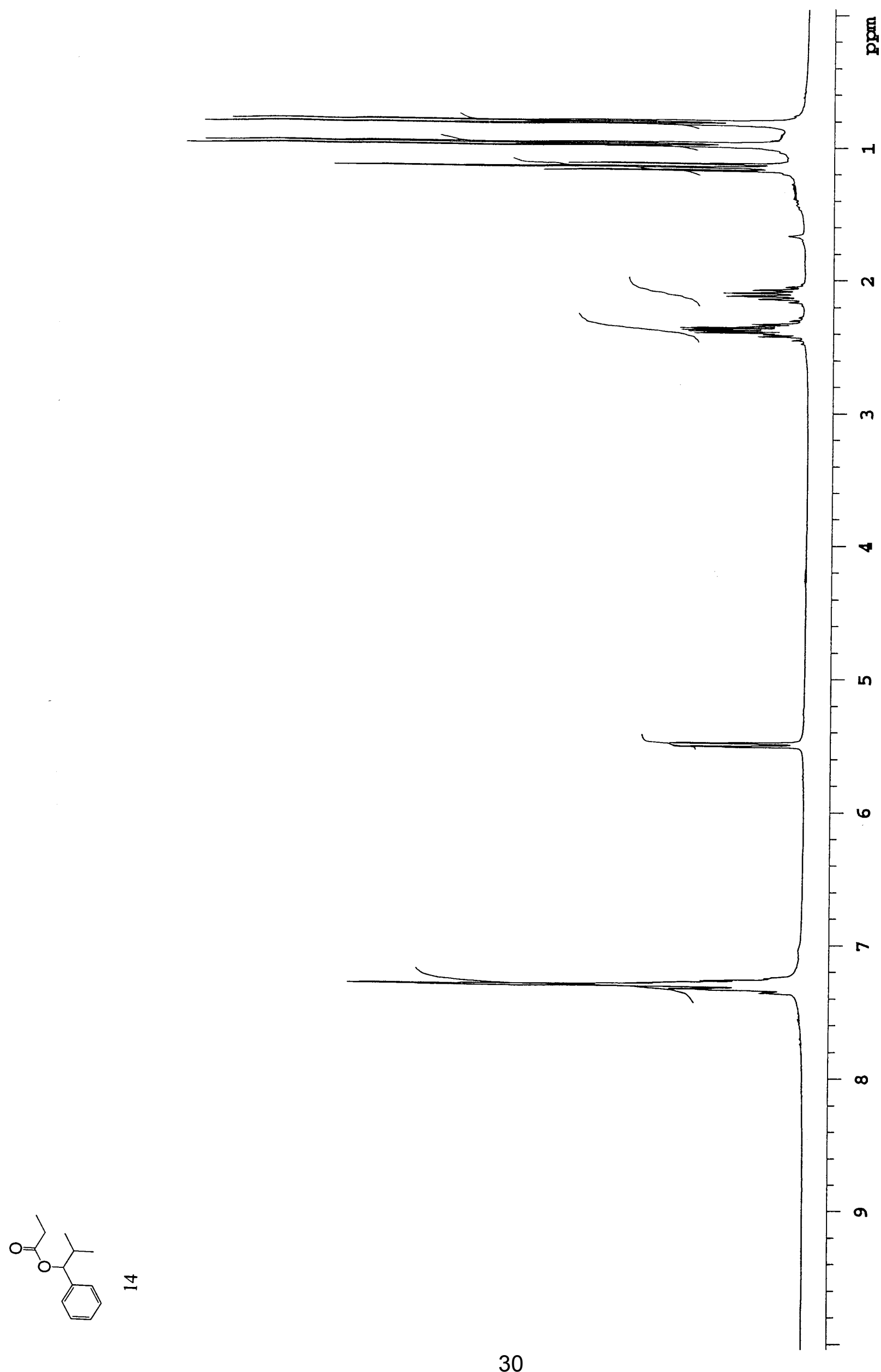




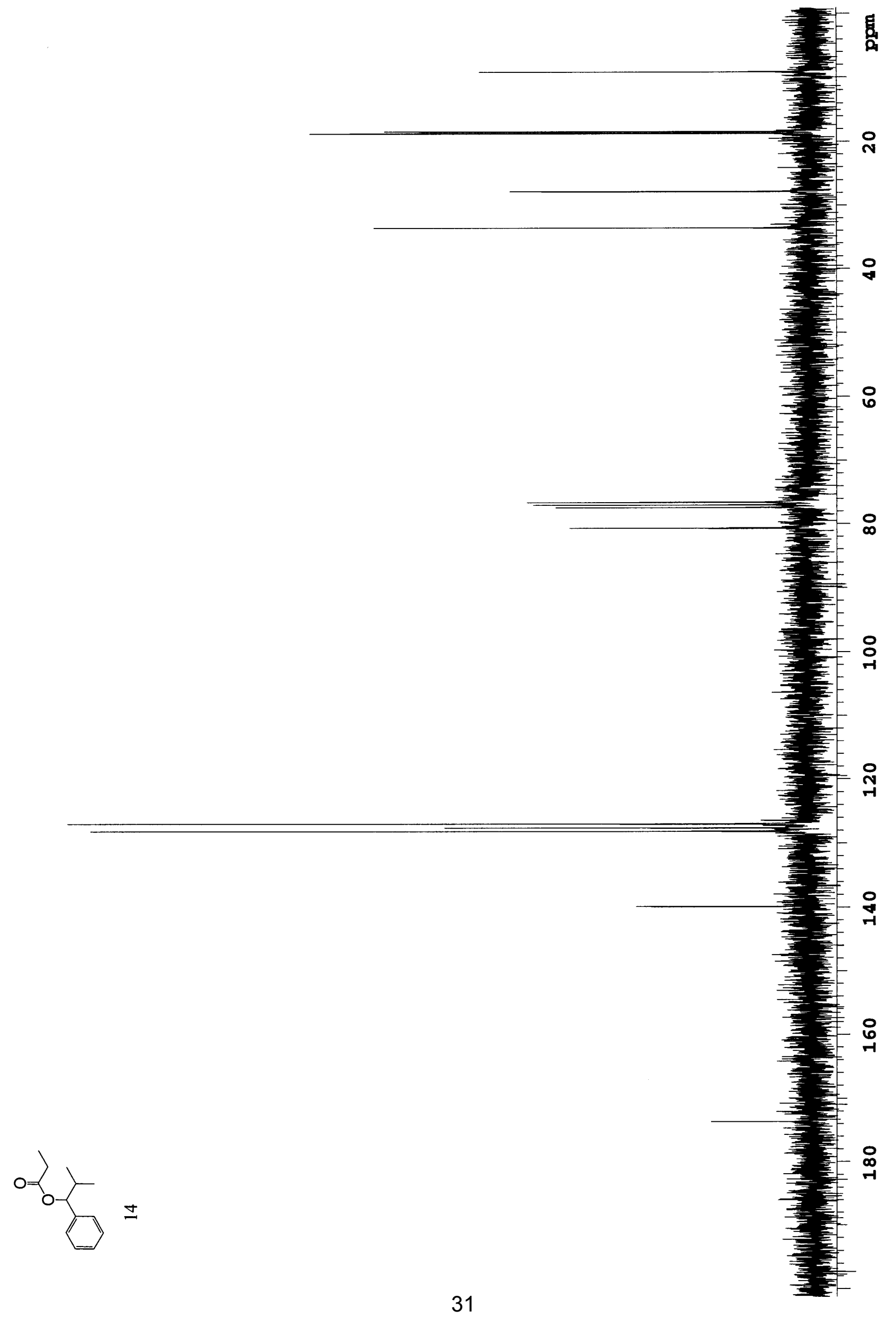




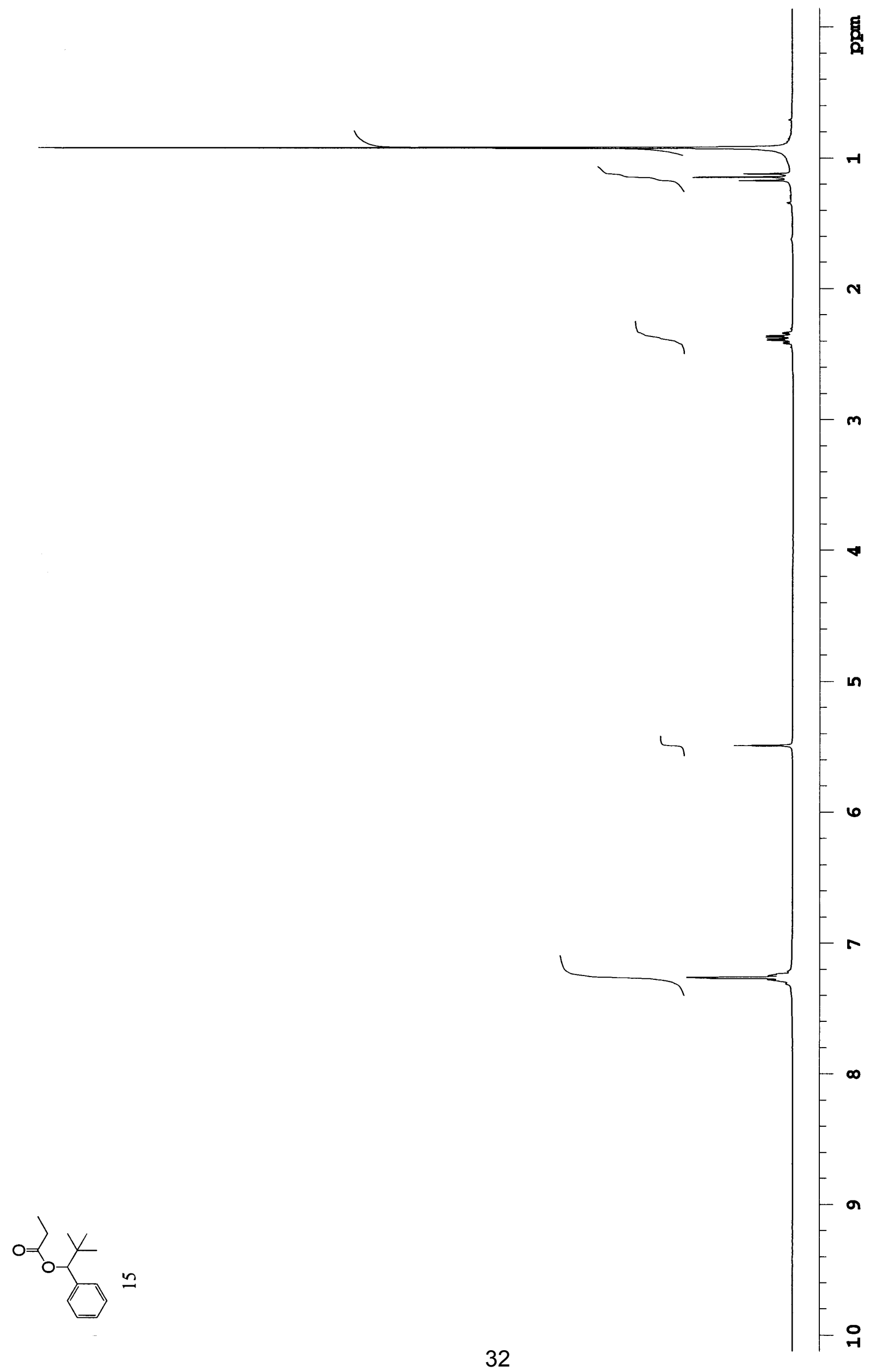




$$
1
$$




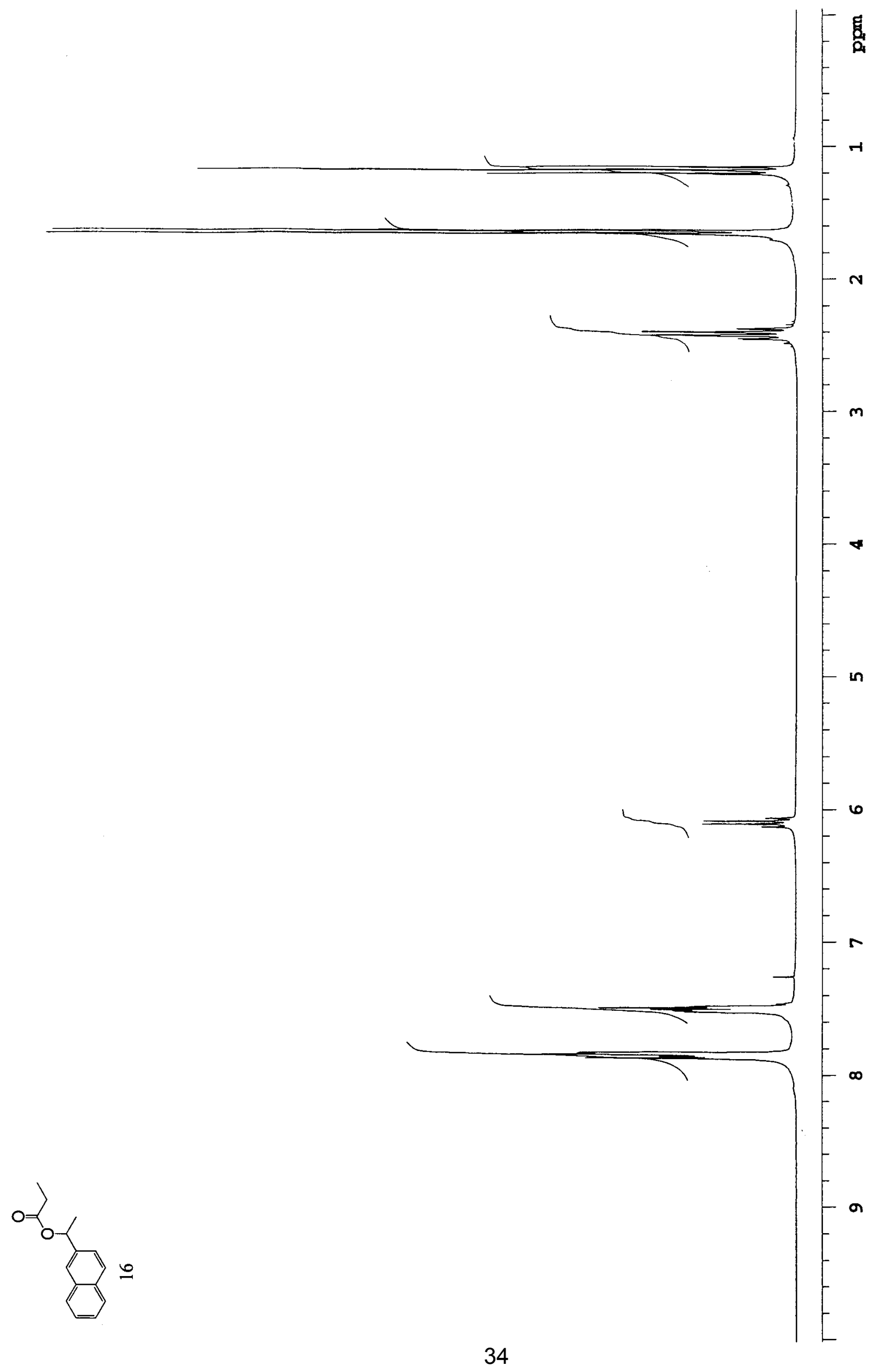




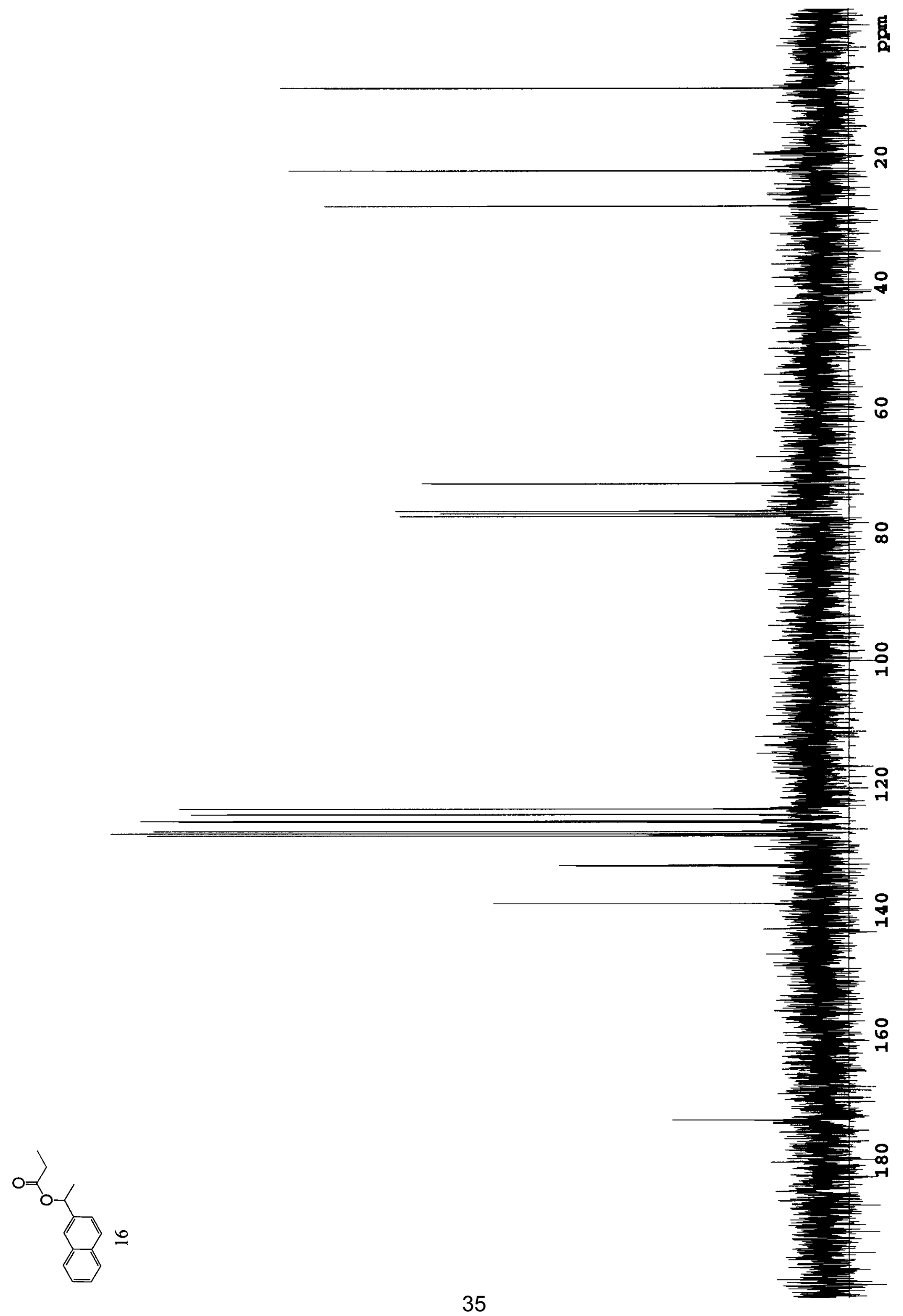




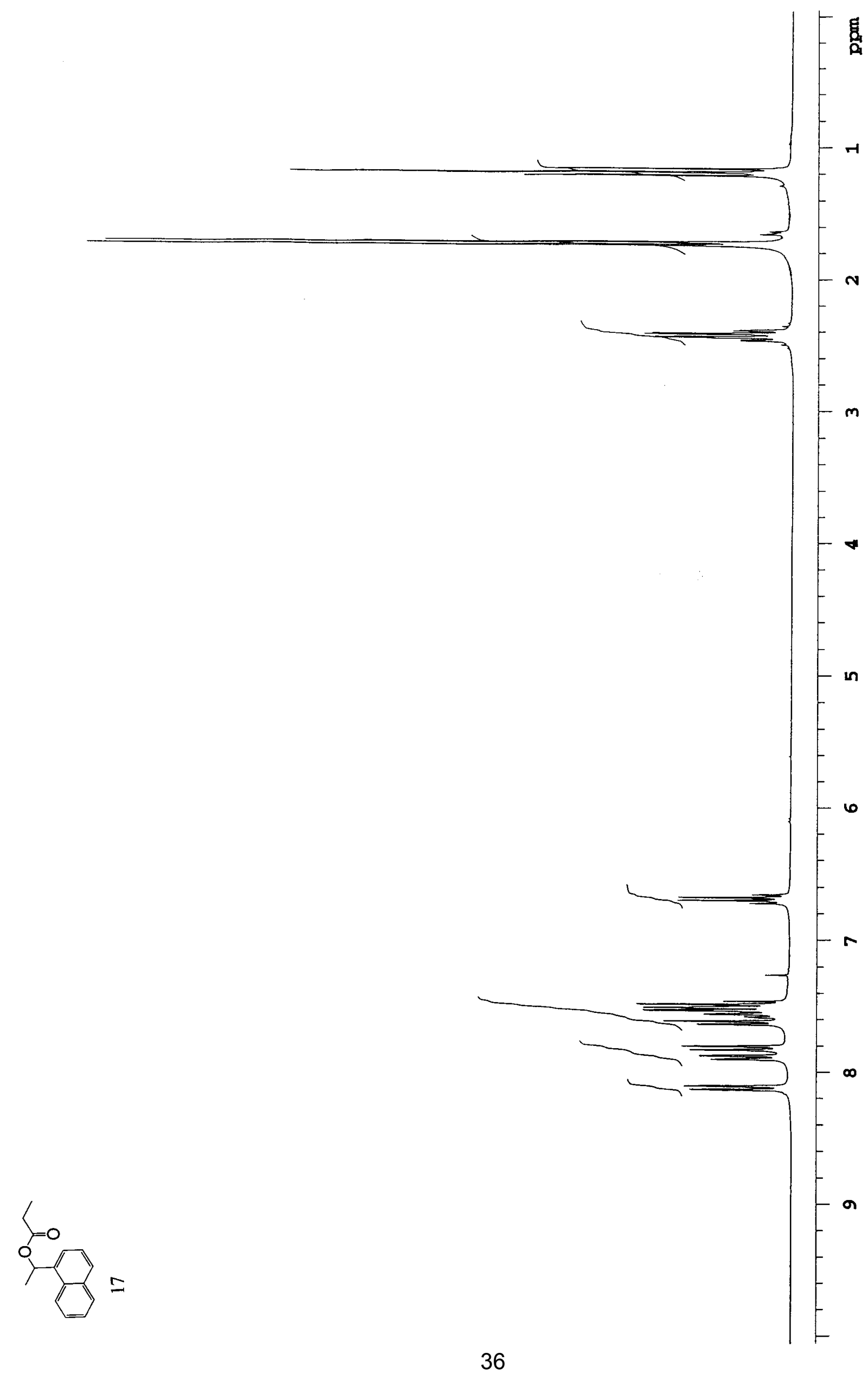




$$
4
$$




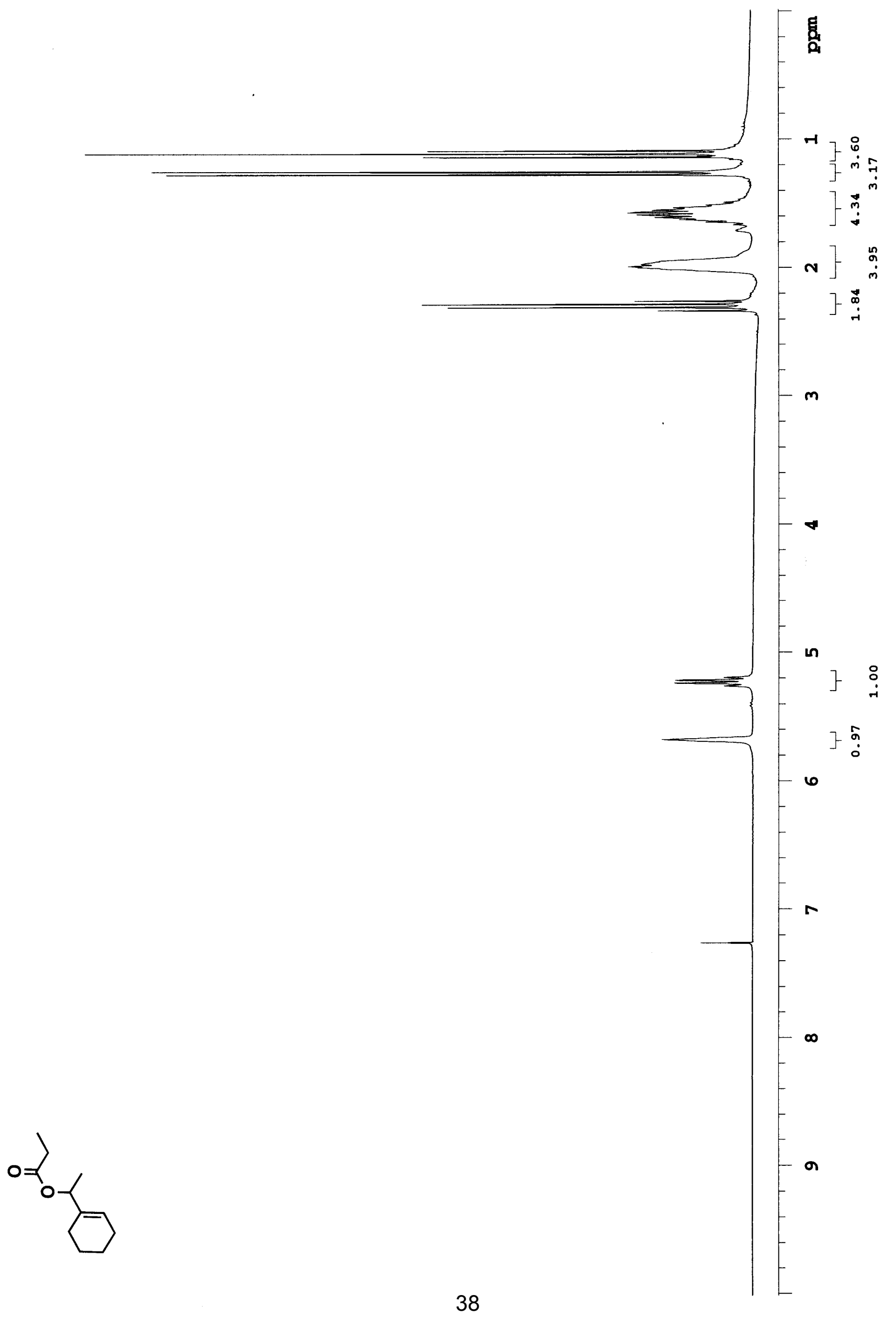




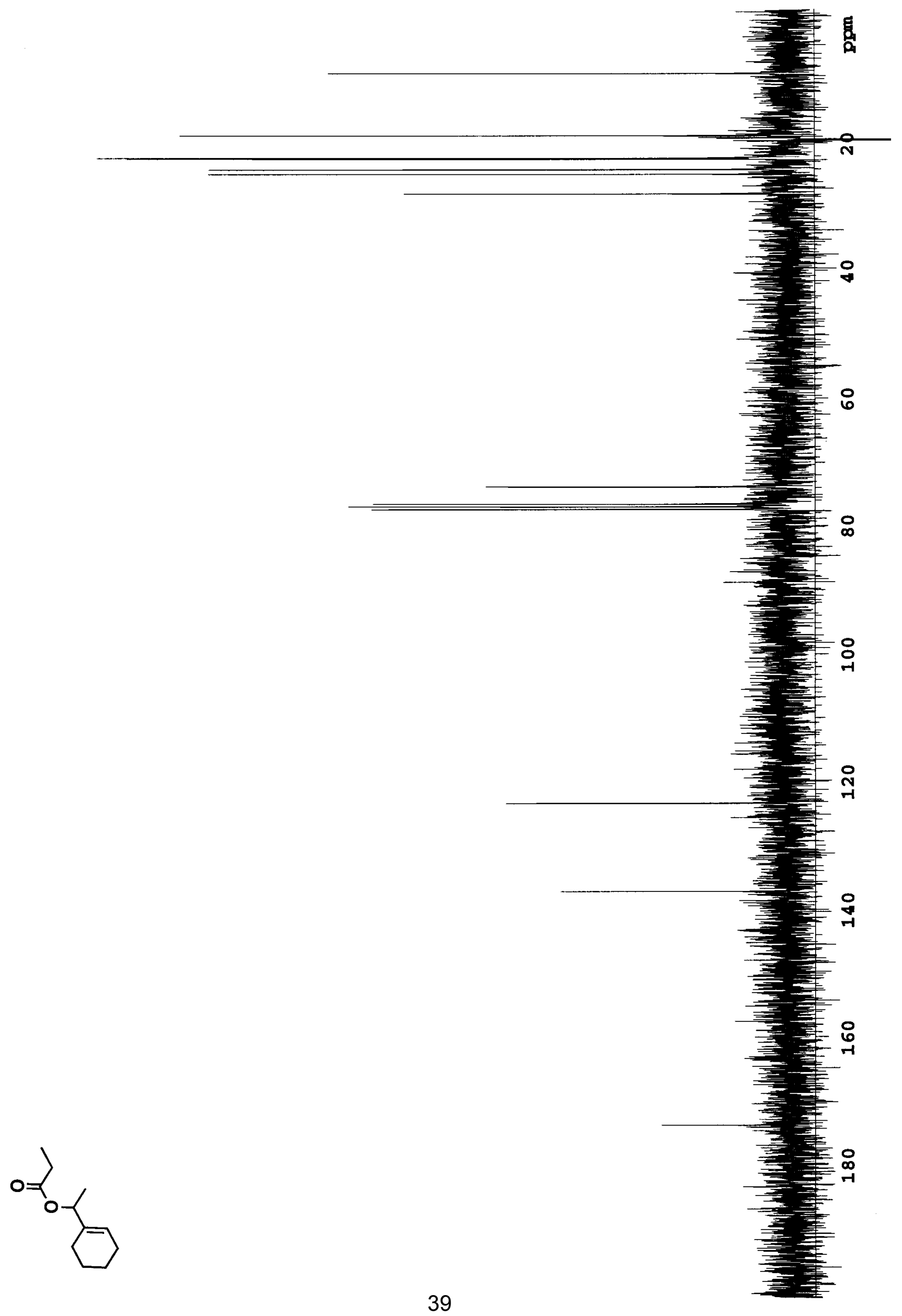




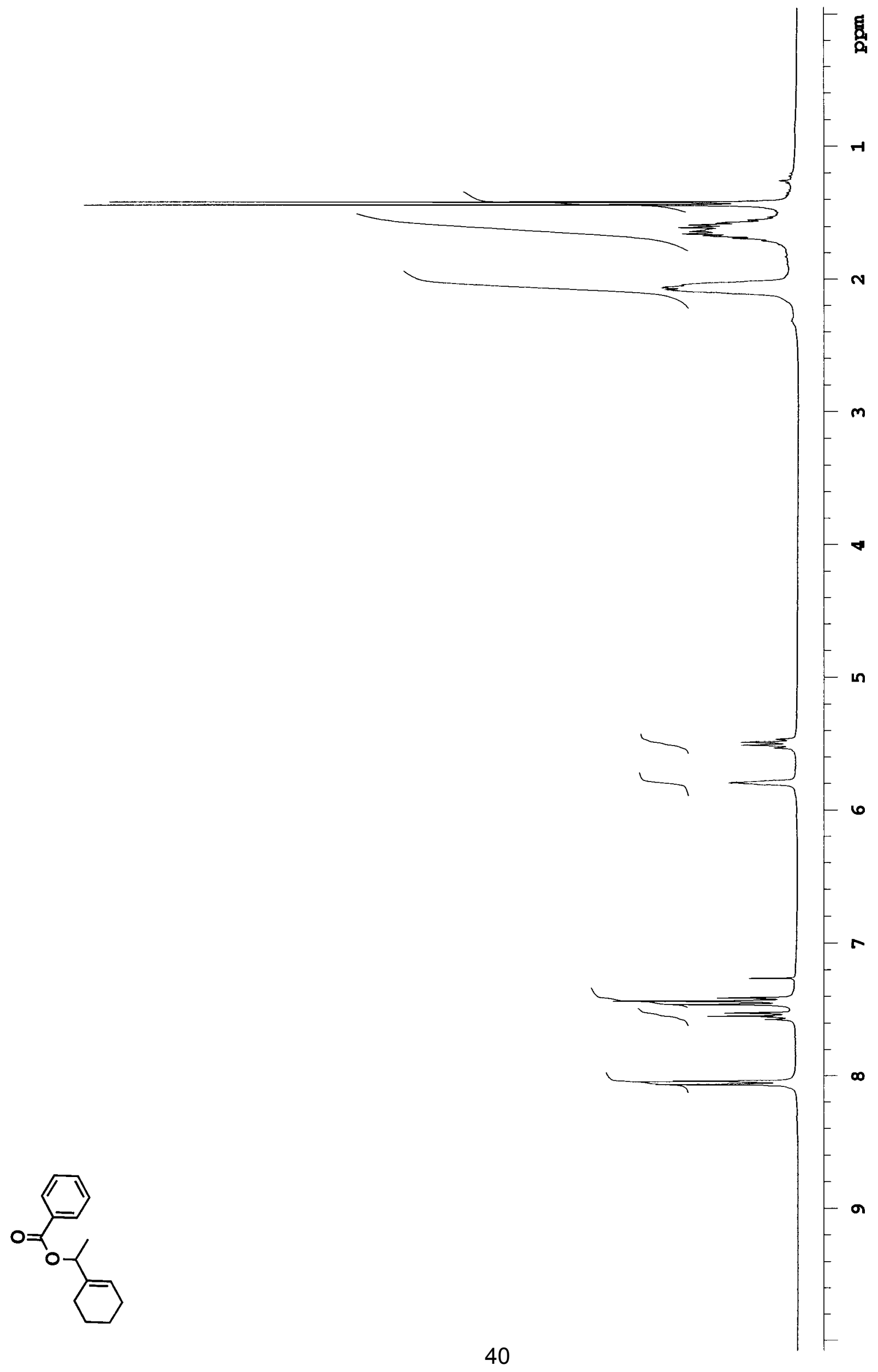




$$
1
$$

